\title{
Stringy information and black holes
}

\author{
Amit Giveon ${ }^{a}$ and Nissan Itzhaki ${ }^{b}$ \\ ${ }^{a}$ Racah Institute of Physics, The Hebrew University, \\ Jerusalem 91904, Israel \\ ${ }^{b}$ Physics Department, Tel-Aviv University, \\ Ramat-Aviv, 69978, Israel \\ E-mail: giveon@mail.huji.ac.il, nitzhaki@post.tau.ac.il
}

ABSTRACT: We show that in string theory, due to non-perturbative effects, there are cases in which two states that semi-classically are completely different, are in fact the same. One state cannot be excited without exciting the other; they are two components of the same state in the exact theory. As a result, in some situations that include black holes, the nature of information in string theory is dramatically different than in field theory. In particular, each general-relativity state, that lives in the atmosphere of black fivebranes, is accompanied with an excitation that lives on folded strings, which fill the black-hole interior. This is likely related to the way that information is extracted from black holes in string theory, and we refer to it as stringy information.

Keywords: Black Holes in String Theory, Conformal Field Models in String Theory

ARXiv ePrint: 1912.06538 


\section{Contents}

1 Introduction and summary 1

$\begin{array}{lll}1.1 & \text { Outline of the paper } & 6\end{array}$

$\begin{array}{lll}2 & \text { Black fivebranes } & 7\end{array}$

3 Euclidean hints $\quad 10$

$\begin{array}{lll}3.1 & \text { Stringy information and the generalized FZZ duality } & 10\end{array}$

3.2 The $k=1$ transition $\quad 12$

$\begin{array}{lll}3.3 & \text { The fusion } F \sim W^{+} * W^{-} & 14\end{array}$

3.4 The fusion $F_{\ell} \sim W * W_{\ell}^{*} \quad 16$

$3.5 F=I^{k}$ and $\mathrm{BH}=\mathrm{GC} \quad 18$

4 Lorentzian physics $\quad 18$

$\begin{array}{lll}4.1 & I \text { and } W & 19\end{array}$

$4.2 F$, folded string and EPR 20

$4.3 k=1$ and the $\mathrm{BH}$-string transition 22

$5 \quad \mathrm{BH}$ and stringy information 23

$\begin{array}{llr}6 & \text { Discussion } & 27\end{array}$

A $I_{\ell, \bar{\ell}}, W_{\ell, \bar{\ell}}$ and $F_{\ell, \bar{\ell}}$

В $\beta^{\ell}=-P_{\ell}(\partial w, \ldots) \quad 28$

$\begin{array}{ll}\text { C Folded string from } \boldsymbol{A d S}_{3} & 29\end{array}$

D The construction of $W_{V}$

E Some facts on $\operatorname{SL}(2, \mathbb{R}) / U(1) \quad 33$

\section{Introduction and summary}

Very loosely speaking, there are two scenarios, both of which can be traced to the work of 't Hooft from about 30 years ago $[1,2]$, that prevent black holes from destroying information. The brutal and gentle scenarios. In the brutal scenario, there is a mechanism, yet to be found, that prevents information from falling into the black hole $(\mathrm{BH})$. In the gentle scenario, information can fall into the $\mathrm{BH}$, but it is somehow also encoded outside the BH.

Being the leading candidate for a theory of quantum gravity, string theory is expected to indicate which way to go. So far, we have learned from string theory, via the AdS/CFT 
correspondence [3], that BHs cannot destroy information, but we still do not understand why - what is the mechanism that encodes the information in the radiation? Is it brutal or gentle? The reason why string theory fails to provide an answer so far is that, in general, we expect non-perturbative effects in the Newton constant, $G_{N} \sim g_{s}^{2}$, where $g_{s}$ is the string coupling, to play a key role in resolving the $\mathrm{BH}$ information puzzle, and these are hard to describe in string theory.

Black fivebranes are interesting in that regard, since there is an exact worldsheet conformal field theory (CFT) description of their near horizon regime. As a result, nonperturbative effects in $\alpha^{\prime}=l_{s}^{2}$, where $l_{s}$ is the string length scale, are well understood. The exactness of the CFT is useful only for small $g_{s}$, in which case $l_{p} \ll l_{s}$, where $l_{p}$ is the Planck length scale. Hence, generally speaking, one would expect the physics associated with the non-perturbative effects in $l_{s}$ to be quite different than the non-perturbative effects in $l_{p}$. Still, since perturbative effects in $\alpha^{\prime}$ generate similar terms in the effective action as perturbative effects in $G_{N}$, it is natural to wonder if non-perturbative $\alpha^{\prime}$ effects can teach us something useful about the $\mathrm{BH}$ information puzzle.

In this paper, we argue that they do. We show that, at least in the case of black fivebranes, non-perturbative effects modify the nature of information in string theory in a rather dramatic fashion; two states that semi-classically are completely different, might actually be the same in string theory. More precisely, they are two components of the same state, which in practice means that they cannot be excited separately, but only simultaneously. In particular, we show that each of the ordinary general relativity (GR) modes, that propagates in the $\mathrm{BH}$ atmosphere, has a partner in the $\mathrm{BH}$ interior. If the GR mode is excited then the partner must be excited too. This is in the spirit of $A=R_{B}[4,5]$ and the $\mathrm{ER}=\mathrm{EPR}[6]$ proposals, which suggest a gentle scenario. However, as we shall see, things are more complicated.

What allows us to make such a precise identification is the fact that the stringy setup that we inspect is that of near near-extremal black fivebranes, e.g. those corresponding to a stack of $k$ Neveu-Schwarz (NS) fivebranes. String theory on this background is described by an exact two-dimensional CFT. In particular, the physics in the radial and time directions is described by the coset $\mathrm{SL}(2, \mathbb{R})_{k} / \mathrm{U}(1)$ CFT [7-11]. This coset description led to some exact results, that manage to sum up all non-perturbative effects in $l_{s}$, on the sphere [12], and in some cases on the torus [13].

The target-space interpretation of these non-perturbative effects turned out to be quite non-trivial even for large $k$, when at least naively, $\alpha^{\prime}$ corrections are expected to be negligible (see e.g. [14-18], for a review). For example, the target-space interpretation of the exact reflection coefficient, [12], is quite surprising in a rather transparent way, [14, 19], in the Euclidean $\mathrm{BH}$ case - the cigar geometry, and in a more subtle way, [18, 20], in the Lorentzian case.

The relation between the non-perturbative effects in $\alpha^{\prime}$ and stringy information can be traced all the way to the FZZ duality [21, 22]. The target-space interpretation of the FZZ duality is the following. We start with a cylinder background $S^{1} \times R_{\phi}$, where $R_{\phi}$ is a spatial direction with a linear dilaton, $\Phi(\phi)=-\frac{1}{2} Q \phi$, and the radius of the $S^{1}$ is 
$2 / Q \cdot{ }^{1}$ The strong coupling region, at $\phi \rightarrow-\infty$, can be chopped of by condensing an operator, that we refer to as $I$, that turns the cylinder into the cigar $\operatorname{SL}(2, \mathbb{R})_{k} / \mathrm{U}(1) \mathrm{CFT}$ background (with $k=2 / Q^{2}$ ). ${ }^{2}$ The condensate of $I$ determines the string coupling at the tip, $g_{0}$. The bigger the condensate is the smaller $g_{0}$ is. $I$ is a truly marginal operator and the target-space interpretation of this is that the free energy associated with this background vanishes. FZZ duality implies that the condensation of $I$ is accompanied with a condensation of a completely different operator — the sine-Liouville operator — which we denote by $W=W^{+}+W^{-}$. The reason for this notation is that from a stringy point of view, the sine-Liouville operator is a linear combination of a string that winds the $S^{1}$ with a winding number $\omega=1$ and its conjugate one, with $\omega=-1$. Regardless of the orientation of the string, its energy is minimized at the tip, and so the wave function of the sine-Liouville operator is heavily suppressed away from the tip.

The target-space interpretation of the FZZ duality is fascinating. At large $\phi$, the wave functions of $I$ and $W^{ \pm}$are very different: $I \sim \exp (-Q \phi) \simeq g_{s}^{2}(\phi)$ while $W^{ \pm} \sim \exp (-\phi / Q)$. Still, the FZZ duality implies that they amount to the same state or, more precisely, two components of the same state. The two are tied together by the boundary condition at the tip [23]. From the underlying $\mathrm{SL}(2, \mathbb{R})$ perspective, the origin of the FZZ duality is an isomorphism of representations [15]. This implies that the FZZ duality can be generalized to many other pairs of states. In fact, in [15] it was shown that all the states that live at the tip of the cigar ${ }^{3}$ - with a wave function that scales like $\exp \left[\left(-\frac{1}{Q}+(\ell-1) Q\right) \phi\right]$ $(\ell=1,2, \ldots)$ - have a partner that lives at the cap of the cigar - with a wave function that scales like $\exp (-Q \ell \phi)$, respectively. An observer that has access only to large $\phi$ will think that these must be two separated states that for some reason get excited simultaneously. An observer that has access to the tip of the cigar will realize that these are two components of the same state, which are linked by the boundary condition at the tip.

Upon analytic continuation, the tip of the cigar is mapped to the horizon of the $\mathrm{BH}$ and the cap of the cigar to the atmosphere of the BH, where most Hawking quanta live before they escape to infinity. Hence, it is natural to wonder if a Lorentzian version of the Generalized FZZ (GFZZ) duality exists and if it gives a precise realization of $A=R_{B}$ and/or $E R=E P R$. There is, however, a basic problem with the analytic continuation of the FZZ duality. The analytic continuation of $W^{ \pm}$is not mutually local with vertex operators, $V_{E}$, that are associated with ordinary states that propagate in the $\mathrm{BH}$ geometry and carry some finite energy, $E$.

Only very recently, a way out was proposed [24]. From the point of view of the underlying $\mathrm{SL}(2, \mathbb{R})$ theory, the reason why the truly marginal operators $I$ and $W$ condense is that they are invariant under the $\mathrm{SL}(2, \mathbb{R})_{L} \times \mathrm{SL}(2, \mathbb{R})_{R}$ current algebra; they are screening operators. Simply put, their condensation does not break any of the symmetries. It is known for many years that there is yet another truly marginal operator, denoted by $F$, that does not break the $\mathrm{SL}(2, \mathbb{R})_{L} \times \mathrm{SL}(2, \mathbb{R})_{R}$ current algebra $[25,26] . F$ is interesting

\footnotetext{
${ }^{1}$ We set $\alpha^{\prime}=2$ here.

${ }^{2}$ In the supersymmetric case, which we discuss here, it amounts to the continuation of the black fivebranes above to Euclidean space-time.

${ }^{3}$ We discuss the cigar with a large curvature length, $\sqrt{\alpha^{\prime} k}$, a.k.a. a parametrically small $Q$.
} 


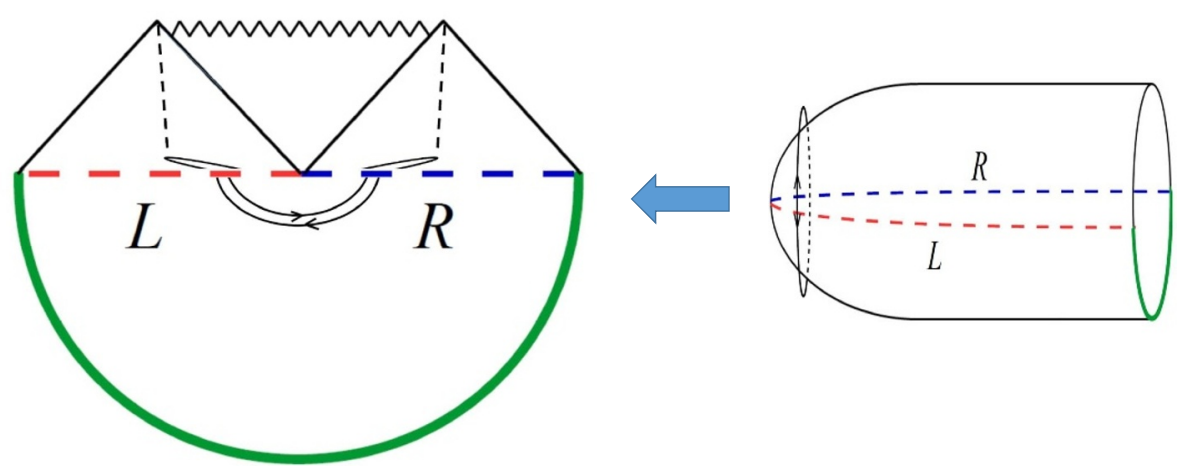

Figure 1. The target-space interpretation of the CFT fusion (1.1): in the Euclidean section (on the right), the cigar background is accompanied, due to the FZZ duality, with a condensate of winding $\omega= \pm 1$ strings, denoted in the text by $W^{ \pm}$. As usual, the Lorentzian section is obtained by cutting the cigar and analytically continue. When doing so, $W^{ \pm}$are combined to form a folded string, that folds towards the $\mathrm{BH}$.

since, unlike $W^{ \pm}$, it is mutually local with $V_{E}$ and so it can condense also in the BH case. $F$, however, appears to be problematic since it is well defined only for integer $k$, and it is outside the unitarity bound. This is the reason why to a large extent $F$ was ignored. Nevertheless, in a series of papers [27-30], it was shown that a condensation of the formal analytic continuation of $F$ to any $k$ describes the same non-perturbative physics in $\alpha^{\prime}$ as the condensation of $W=W^{+}+W^{-}$.

In [24], it was proposed that the success of $[27,28]$ is not accidental. It follows from the fact that $F$ is a fusion of $W^{+}$with a $W^{-}$, schematically,

$$
F \sim W^{+} * W^{-}
$$

namely, $F$ is a bound state of $W^{+}$and $W^{-}$. The calculations done in $[27,28]$, with $F$ as a screening operator, give the same results as the "correct calculations," [29, 30], with $W^{+}+W^{-}$as a screening operator, since the contribution comes from the points where $W^{+}$and $W^{-}$coincide.

The target-space interpretation of (1.1) is in the spirit of the Hartle-Hawking wave function [31], but with a stringy twist. The latter involves folded strings, which were proposed in our context in [32,33], and are related to those in [34-36]. Concretely, in the Euclidean section, $W^{+}+W^{-}$condenses. In the Lorentzian section, $W^{+}$and $W^{-}$are glued together to form a folded string, $F$ [24]; see figure 1. The upshot is that the eternal $\mathrm{BH}$ geometry is accompanied with folded strings that are described by a condensation of $F$. Just like in the FZZ duality, the eternal BH geometry and the folded strings are two components, that from the target-space point of view look very different, of the same state.

The relation with $\mathrm{ER}=\mathrm{EPR}$ can be made more precise. Starting with the Euclidean cylinder and following the Hartle-Hawking procedure, we get two disconnected flat spacetimes. Condensation of $I$ connects them, by forming an ER bridge. On the other hand, condensation of $F$ does something quite different. $F$ is the operator that creates a folded 
string. The folded string lives on both space-times. Consequently, a condensation of $F$ entangles the two space-times. A condensation of $F$, therefore, realizes EPR.

This can be extended to include excitations that propagate in the $\mathrm{BH}$ atmosphere. Such states are accompanied by localized dual modes, which live on the folded string, in the $\mathrm{BH}$ interior. Again, the two are different components of the same state. One cannot have an excitation in the $\mathrm{BH}$ atmosphere without the mode that excites the folded string. And, again, this follows from isomorphism of $\mathrm{SL}(2, \mathbb{R})$ representations. The details are described in the bulk of the paper; here, we sketch the way this works.

Consider a massless excitation that propagates in the $\mathrm{SL}(2, \mathbb{R}) / \mathrm{U}(1) \mathrm{BH}$ geometry using the tortoise coordinate, $r^{*}$. The potential such an excitation experiences vanishes at the horizon, $r^{*} \rightarrow-\infty$, and goes to a constant at infinity, $r^{*} \rightarrow \infty$. The constant is induced by the linear dilaton and it scales like $Q^{2} \sim 1 / k$. The potential is monotonic with a small mass gap. From the underlying $\operatorname{SL}(2, \mathbb{R})$ point of view, the excitations above the gap, $E_{s}>Q^{2}$ (where $E_{s} \sim E^{2}$ is the energy in the relevant Schrodinger equation and $E$ is the energy with respect to the Schwarzschild time), that can escape all the way to $r^{*}=\infty$, are in the principal continuous representations. As such, they neither have a GFZZ dual, nor an interior dual.

However, the excitations below the gap, $0<E_{s}<Q^{2}$, arise from the principal discrete representations of the underlying $\operatorname{SL}(2, \mathbb{R})$. This is crucial, and at first sight confusing. It is confusing since the energy in the range $0<E_{s}<Q^{2}$ is continuous, still these states are reduced from the discrete representations. This is crucial, since states in the discrete representations have GFZZ duals. The GFZZ duals involve spectral flow in the compact, time-like $J^{3}$ direction, while $E$ is the energy with respect to the non-compact, space-like $J^{2}$ direction. As a result, the details of the GFZZ duals are more subtle, since they involve a change from the standard basis, of $J^{3}$ eigenstates, to the hyperbolic one of $J^{2}$ eigenstatses. The end result of this procedure is that all the standard states that live in the $\mathrm{BH}$ atmosphere are accompanied with a partner that lives on the folded string, in the $\mathrm{BH}$ interior, with a tail outside the $\mathrm{BH}$, localized a distance of order the string length scale near the horizon, and whose wave function scales like ${ }^{4} \exp (-2 \phi / Q)$, asymptotically in the radial direction.

All of this seems to fit neatly with ER=EPR and/or $A=R_{B}$. There is, however, a twist in the story. The original motivation for $\mathrm{ER}=\mathrm{EPR}$ and $A=R_{B}$ was to evade the firewall paradox [37] (for earlier claims that a unitary BH evolution must result in a singular horizon, see e.g. [38-40]) and to extract the information in a gentle fashion. While the black fivebrane in string theory seems to give a precise realization of the $\mathrm{ER}=\mathrm{EPR}$ conjecture, it is far from being clear that it implies a smooth horizon, nor a fuzzy one [41, 42]. After all, the fact that $I$ is accompanied with $F$ implies that an infalling observer will encounter the folded strings and, at least classically, their energy-momentum tensor seems to imply a singular horizon [33].

Put differently, schematically, the difference between the black fivebranes and $\mathrm{ER}=\mathrm{EPR}$ is the following. Indeed, $I$ and $F$ are the operators that correspond to ER

\footnotetext{
${ }^{4}$ For parametrically small $Q$, a.k.a for a large BH.
} 
and EPR, respectively. However, the statement is not that $I=F$. Such a statement would mean that we can either condense $I$ or condense $F$ and get the same physics. But this is not the case. For example, the condensation of $I$ is responsible for poles in some correlation functions at imaginary momentum $p \sim i Q n$ (with integer $n$ ), while the condensation of $F$ generates poles in the same correlation functions at $p \sim i n / Q$, [43]. Therefore, both $I$ and $F$ condense, in an unambiguous way [28]. The condensation of $I+F$ means that the ER bridge is not empty, but is filled with folded strings, which are responsible for the entanglement of the left and right wedges of the eternal $\mathrm{BH}$.

Needless to say that the fate of an infalling observer in this setup deserves more serious considerations. In particular, it is possible that finite $g_{s}$ effects could render the horizon smooth or fuzzy. This is possible even at arbitrarily small $g_{s}$, since the number of folded strings is expected to scale like $1 / g_{s}^{2}$, [33].

It turns out that this stringy setup also resonates with the idea that the $\mathrm{BH}$ interior is made out of a condensate of gravitons [44, 45]. As discussed above, in the near-extremal NS fivebranes case, the $\mathrm{BH}$ interior is made out of folded strings. However, the operator $F$, the screening operator that corresponds to the condensation of the folded string, looks far from the $\mathrm{BH}$ like a product of $k$ gravitons: schematically,

$$
F=I^{k}
$$

where $I$ is the screening operator that creates the gravitational background. More precisely, the gravitons bound state dual of the folded string condensate is the fusion of $k$ gravitons condensates. We shall refer to such a duality between the $\mathrm{BH}$ interior and the bound state of gravitons condensate as $\mathrm{BH}=\mathrm{GC}$.

Finally, the black fivebranes also realize the correspondence between BHs and fundamental strings, [46-48]. The Euclidean version of this was discussed sometime ago [49, 50]. Here, we discuss the Lorentzian case, and emphasis the fact that there is a twist in the plot here too.

\subsection{Outline of the paper}

In the next section, we review some of the properties of black fivebranes. An important feature of the relevant worldsheet background, near solitonic black fivebranes, is that it amounts to perturbative string theory on a two-dimensional black hole $(\mathrm{BH})$, which is described by an exact worldsheet Conformal Field Theory $(\mathrm{CFT})$ - the $\mathrm{SL}(2, \mathbb{R})_{k} / \mathrm{U}(1)$ coset CFT, where $k$ is the fivebranes charge. Hence, one may reveal exact stringy aspects of the theory, including those that are non-perturbative in the string scale, $\alpha^{\prime}=l_{s}^{2}$.

In section 3 , we collect some hints about the nature of stringy information, from considering the simpler case of string theory on the Euclidean version of the black hole - the cigar, worldsheet CFT. We start by reviewing in subsection 3.1 the GFZZ duality. We define the GR-like modes $I_{\ell, \bar{\ell}}$, their stringy duals $W_{\ell, \bar{\ell}}$, and discuss the reasoning that leads to the conclusion that they are two components of the same state in the theory. The FZZ duality, which corresponds to $\ell=\bar{\ell}=1$, is used in subsection 3.2 to discuss the Euclidean $\mathrm{BH}$-strings transition, that takes place when the size of the $\mathrm{BH}$ is $\ell_{s}$ (at $k=1$ ). 
In subsection 3.3, the definition of $F$ via the fusion (1.1), and some of its consequences, are discussed. In subsection 3.4, we combine the GFZZ duality with the fusion to describe excitations of $F$, that schematically take the form

$$
F_{\ell, \bar{\ell}} \sim W * W_{\ell, \bar{\ell}}^{*} .
$$

The GR modes, $I_{\ell, \bar{\ell}}$, and their non-perturbative stringy completion, $F_{\ell, \bar{\ell}}$, can be viewed as the stringy Euclidean version of ER=EPR. In subsection 3.5, we discuss (1.2), and its possible relation with a $\mathrm{BH}=\mathrm{GC}[44,45]$.

In section 4, we turn to the actual $\mathrm{BH}$. We start by discussing in subsection 4.1 the subtleties with the analytic continuation of the winding string condensate, $W$, to Lorentzian signature. In subsection 4.2 , we present the target-space interpretation of the condensate $F$ - the continuation of the fusion (1.1) to Lorentzian space-time - as a BH-filling folded string, and the light it sheds on the BH-string transition is discussed in subsection 4.3.

We also argue in section 4 that the folded string entangles the two sides of the eternal $\mathrm{BH}$. This is made more precise in section 5, where excitations of the $\mathrm{BH}$ are considered. It is shown that a GR mode that propagates, say, in the $\mathrm{BH}$ atmosphere of the right wedge, has a non-perturbative stringy completion, in the form of an excitation of the $\mathrm{BH}$ interior-filling folded strings, with tails on both sides of the eternal BH.

Finally, section 6 is devoted for a discussion, and various technical details are collected in a few appendices.

\section{Black fivebranes}

In this section, we describe the ten-dimensional black-brane geometry, corresponding to Neveu-Schwarz (NS) solitonic branes, with a finite energy density above extremality, in type II superstring theory, their near horizon limit, that includes the two-dimensional black hole, and its powerful description in terms of an exact worldsheet CFT.

The string-frame geometry of $k$ coincident near extremal NS fivebranes in the type II superstring is, [51],

$$
\begin{aligned}
& d s^{2}=-\left(1-\frac{r_{0}^{2}}{r^{2}}\right) d t^{2}+\left(1+\frac{k \alpha^{\prime}}{r^{2}}\right)\left(\frac{d r^{2}}{1-\frac{r_{0}^{2}}{r^{2}}}+r^{2} d \Omega_{3}^{2}\right)+\left(d y_{1}^{2}+\cdots+d y_{5}^{2}\right), \\
& e^{2 \Phi}=g^{2}\left(1+\frac{k \alpha^{\prime}}{r^{2}}\right),
\end{aligned}
$$

where $\Phi$ is the dialton field, and there is an $H$ flux with $k$ units on the three sphere $d \Omega_{3}^{2}$, that we did not write, since it will not play any role below. This background describes black fivebranes with mass $M$ per unit five volume $V_{5}$,

$$
\frac{M}{V_{5}}=\frac{M_{s}^{6}}{(2 \pi)^{5}}\left(\frac{k}{g^{2}}+\mu\right)
$$

where

$$
\mu=\frac{r_{0}^{2} M_{s}^{2}}{g^{2}}
$$


$\mu /(2 \pi)^{5}$ is the energy density above extremality in string units and $M_{s}$ is the string mass scale, $M_{s}=1 / l_{s}=1 / \sqrt{\alpha^{\prime}} .5$

We are interested in the physics near the horizon of the black fivebranes. The nearhorizon geometry is obtained [52] by taking the asymptotic string coupling $g$ to zero, $g \rightarrow 0$, while keeping the energy density $\mu$ finite, namely, $r_{0} \sim g l_{s}$ in the limit. Introducing the coordinate $\phi$, related to the radial coordinate $r$ by

$$
r=r_{0} \cosh \left(\frac{\phi}{\sqrt{2 k}}\right)
$$

one finds [52] the geometry (from here, we work with $\alpha^{\prime}=2$ )

$$
d s^{2}=-\tanh ^{2}\left(\frac{\phi}{\sqrt{2 k}}\right) d t^{2}+d \phi^{2}+2 k d \Omega_{3}^{2}+\left(d y_{1}^{2}+\cdots+d y_{5}^{2}\right),
$$

and dilaton

$$
e^{2 \Phi}=\frac{g_{0}^{2}}{\cosh ^{2}\left(\frac{\phi}{\sqrt{2 k}}\right)}, \quad g_{0}^{2}=\frac{k}{\mu},
$$

which describes the exterior of a two-dimensional black hole in the time $t$ and radial $\phi$ directions - a Schwarzschild-like $2 d$ black hole [7-11], with an asymptotically linear dilaton - times a three sphere with radius $\sqrt{2 k}$ and with $k$ units of $H$ flux, times a five torus; $g_{0}$ is the value of the string coupling at the horizon of the black hole, located at $\phi=0$.

We will also be interested to collect hints from properties of the simpler case of string theory on the Euclidean black hole. The latter is obtained from the above background by the analytic continuation $t \rightarrow i x=i \sqrt{2 k} \theta$ (and for some purposes, we may Wick rotate also, say, $y_{5} \rightarrow i \tau$, to establish a string theory on the Euclidean black hole times real time). The two-dimensional black-hole geometry in (2.6) thus turns into a Euclidean cigar-shaped background, [7-11],

$$
d s_{E}^{2}=2 k \tanh ^{2}\left(\frac{\phi}{\sqrt{2 k}}\right) d \theta^{2}+d \phi^{2},
$$

with a dilaton given in (2.7). The angular direction of the cigar, $\theta$, has a standard periodicity, $\theta \sim \theta+2 \pi$, such that the cigar is smooth at its tip, located at $\phi=0$. The asymptotic radius of the cigar is $\sqrt{2 k}$. This means, in particular, that the Hawking temperature of the black hole is

$$
T=\beta^{-1}=(2 \pi \sqrt{2 k})^{-1} .
$$

The regime of size of order $\sqrt{k}$ around the tip is the cap of the cigar; in this regime, the radius of curvature is also of order $\sqrt{k}$. In the Lorentzian case, (2.6), it amounts to the thermal atmosphere of the black hole. On the other hand, the asymptotic regime is a flat cylinder, $\mathbb{R}_{\phi} \times S_{x}^{1}, x \sim x+2 \pi \sqrt{2 k}\left(\mathbb{R}^{1,1}\right.$ in the Lorentzian case), with a linear dilaton

$$
\Phi_{\text {asymptotic }}=-\frac{Q}{2} \phi
$$

\footnotetext{
${ }^{5}$ For a finite energy density (2.4), the size of the black fivebrane is of order the Planck scale, $r_{0} \sim g_{s} l_{s}$. We also take the near-horizon limit. As a result, just like in the standard AdS/CFT case, the final background below has a small curvature, that scales like $1 / k$.
} 
with a slop

$$
Q=\sqrt{\frac{2}{k}}
$$

in terms of the black-brane charge, $k$.

The sigma-model worldsheet theories on both the two-dimensional black-hole background in (2.6), (2.7), and in its Euclidean version, (2.8), (2.7), have very powerful properties that we discuss next. The geometry (2.6), (2.7) is the sigma-model background of an exact worldsheet CFT. Concretely (see e.g. [53], for a review), it is a product of an axial quotient $\mathrm{CFT}$ of $\mathrm{SL}(2, \mathbb{R})$ by its $\mathrm{U}(1)$ subgroup in a non-compact space-like direction, which we denote by the left and right-handed currents $\left(J^{2}, \bar{J}^{2}\right)$ of $\mathrm{SL}(2, \mathbb{R})$, times the CFT on the group manifold $\mathrm{SU}(2)$, times a five-torus, $T^{5}$,

$$
\frac{\mathrm{SL}(2, \mathbb{R})_{k}}{\mathrm{U}(1)} \times \mathrm{SU}(2)_{k} \times T^{5}
$$

where the level $k$ of both the $\mathrm{SL}(2, \mathbb{R})$ and the $\mathrm{SU}(2)$ affine symmetry algebras is the number of fivebranes above. The details of the 'internal' piece, the $\mathcal{N}=\mathrm{SU}(2)_{k} \times T^{5}$ in the particular example (2.12), as well as for generic internal spaces in string theory on $\mathrm{SL}(2, \mathbb{R})_{k} / \mathrm{U}(1) \times \mathcal{N}$, will not play a role in the following, and we shall thus ignore it.

Similarly, the Euclidean black-hole background, (2.8), (2.7), amounts to an exact $\mathrm{SL}(2, \mathbb{R})_{k} / \mathrm{U}(1)$ quotient CFT, obtained by an axial $\mathrm{U}(1)$ gauging of the underlying $\mathrm{SL}(2, \mathbb{R})_{k}$ theory in its compact time-like direction, whose left and right-moving generators we denote by $\left(J^{3}, \bar{J}^{3}\right)$, respectively. In the type II superstring, this worldsheet CFT has an $N=(2,2)$ supersymmetry, and thus the background (2.8), (2.7) does not receive perturbative corrections in $\alpha^{\prime}[54,55]$. There are, however, very significant aspects of the theory, which are non-perturbative in $\alpha^{\prime}$; they amount to a condensate of a string winding on the angular direction of the cigar, and its excitations. These are the heroes of the stringy aspects of the Euclidean black hole. Similarly, their consequences in the Lorentzian case, are the ingredients that modify dramatically the GR approximation near the horizon and in the interior of the two-dimensional black hole in string theory.

In the setup discussed above, $k$ is an integer. However, as mentioned, there are many cases in string theory on $\mathrm{SL}(2, \mathbb{R})_{k} / \mathrm{U}(1) \times \mathcal{N}$, in which generically $k$ is not an integer (see e.g. [56-58]). For example, the near horizon theory of near-extremal I-branes [59], which is built from a stack of $k^{\prime}$ NS fivebranes stretched in (012345) intersecting a stack of $k^{\prime \prime}$ NS fivebranes stretched in (016789), on a circle in $x^{1}$, is described by the superstring on the exact $\frac{\mathrm{SL}(2, \mathbb{R})_{k}}{\mathrm{U}(1)} \times \mathrm{SU}(2)_{k^{\prime}} \times \mathrm{SU}(2)_{k^{\prime \prime}} \times T^{2}\left(\right.$ or $\left.\mathbb{R}^{2}\right)$ worldsheet $\mathrm{CFT}$, with $\frac{1}{k}=\frac{1}{k^{\prime}}+\frac{1}{k^{\prime \prime}}$, [56].

Finally, as discussed in the introduction, the purpose of these notes is to collect aspects of string theory on the $\mathrm{SL}(2, \mathbb{R})_{k} / \mathrm{U}(1)$ black hole and suggest interpretation of their consequences. The main essence of the physics appears already in the bosonic $\operatorname{SL}(2, \mathbb{R}) / \mathrm{U}(1)$ case. Thus, to avoid presenting the somewhat cumbersome tools involved in the fermionic case, we will present most of the facts and ideas in the bosonic coset CFT, though we shall apply their consequences also in the type II superstring context of our ultimate interest, when desired. In the next section, we first collect hints from string theory on the Euclidean black hole, and we shall then turn to the Lorentzian case in section 4. 


\section{Euclidean hints}

The Euclidean bosonic SL $(2, \mathbb{R})_{k_{b}} / \mathrm{U}(1)$ cigar CFT provides some useful hints about stringy information and its relation with $\mathrm{ER}=\mathrm{EPR}, \mathrm{BH}=\mathrm{GC}$ and the $\mathrm{BH}$-string correspondence. Since, technically, this CFT is much simpler than the eternal BH CFT, we review in this section some relevant known facts concerning string theory on the cigar, and add some new ones.

We denote the level of the underlying bosonic $\mathrm{SL}(2, \mathbb{R})$ theory by $k_{b}$, to distinguish it from

$$
k=k_{b}-2,
$$

that appeared previously for the superstring, which is the case we are ultimately interested in. Many of the details leading to these facts already appear in the literature; we shall thus refer to existing details, instead of repeating them, and leave some straightforward calculations, following known manipulations, to the reader.

In the first subsection, we review the GFZZ duality, and in the second subsection, we discuss the transition at $k=1$, which is related to the $\mathrm{BH}$-string correspondence. In the third subsection, we discuss the $F \sim W^{+} * W^{-}$relation, which will play a key role in the Lorentzian case, and in the fourth subsection, we describe its excitations, which play an important role in a Euclidean realization of ER $=\mathrm{EPR}$. Finally, in the last subsection, we discuss the relation $F=I^{k}$, which hints to the $\mathrm{BH}=\mathrm{GC}$ proposal.

\subsection{Stringy information and the generalized FZZ duality}

In this subsection, we briefly review the GFZZ correspondence studied in [15]. We use the notation of that paper, apart from $k$ there being the bosonic level $k_{b}$ here, and refer to explicit equations copied from there.

The duality, that is a generalization of the FZZ duality, identifies states in string theory that semi-classically are very different. On one side of the duality we have ordinary states in GR that are bound to the cap of the cigar, where by "the cap of the cigar," we mean the region where the curvature is of order $1 / k$, and so its size scales like $\sqrt{k}$ and is much larger than the string size. These states are denoted by $I_{\ell, \bar{\ell}}$, with left and right-handed excitation numbers $\ell, \bar{\ell} \in Z$. On the other side of the duality there are stringy modes (that are simply absent in GR) with winding $\omega= \pm 1$, which we denote by $W_{\ell, \bar{\ell}}$. These modes are localized near the tip of the cigar. The precise statement of the duality is that for each $\ell$ and $\bar{\ell}$ there is only one state in the theory and $I_{\ell, \bar{\ell}}$ and $W_{\ell, \bar{\ell}}$ are, semi-classically, two components of the same state.

Most of what follows relies on this unusual claim, so it is worthwhile to recall its status. The GFZZ duality was suggested since $I_{\ell, \bar{\ell}}$ and $W_{\ell, \bar{\ell}}$ are reduced from operators that sit in the same representation of the underlying bosonic $\operatorname{SL}(2, \mathbb{R})$ theory. A priori, it is possible, of course, that there are more than one state with the same quantum numbers in the theory. Nevertheless, the possibility that an excitation of $I$ and its $W$ partner amount to different states is ruled out, e.g. by inspecting the elliptic genus in the supersymmetric extension of the $\mathrm{SL}(2, \mathbb{R}) / \mathrm{U}(1)$ theory [60] (see subsection 7.3 of [15] for more details) and by the direct, non-trivial study of correlation functions $[16,17]$. 
From the GR point of view, what is special about $I_{\ell, \bar{\ell}}$ is that these are the only states that are located at the cap of the cigar at all times. It is related to the fact that they arise from states in the principal discrete representations of the underlying $\operatorname{SL}(2, \mathbb{R})$ theory. There are other states, that come from infinity and spend a finite time at the cap before escaping back to infinity. These states, that from the $\mathrm{SL}(2, \mathbb{R})$ point of view sit in different representations - the principal continuous ones, do not have GFZZ duals.

For simplicity, in the rest of this subsection, we present the left-right symmetric operators,

$$
I_{\ell} \equiv I_{\ell, \ell}, \quad W_{\ell} \equiv W_{\ell, \ell},
$$

and describe them in some detail. For completeness, the generic $I_{\ell, \bar{\ell}}$ and $W_{\ell, \bar{\ell}}$ are presented in appendix A.

Consider the $\mathrm{SL}(2)_{k_{b}} / \mathrm{U}(1)$ cigar CFT. Asymptotically, it is the CFT on the cylinder, $\mathbb{R}_{\phi} \times S_{x}^{1}, x \sim x+2 \pi \sqrt{2 k}$, with a linear dilaton (2.10). Define ${ }^{6}$

$$
w=\frac{1}{Q} \phi-i \sqrt{\frac{k_{b}}{2}} \tilde{x} ; \quad \bar{w}=\frac{1}{Q} \phi+i \sqrt{\frac{k_{b}}{2}} \tilde{x},
$$

where $\tilde{x}=x_{L}-x_{R}$ is the T-dual coordinate to a canonically normalized scalar field, $x=x_{L}+x_{R}$, and the background charge $Q$ of $\phi$ is related to the 'total level' $k$ in (3.1) as in section $2,(2.11)$.

The operators $I_{\ell}$, that generate the GR bound states in the cigar CFT, are described asymptotically by the operators in eq. (5.13) of [15],

$$
I_{\ell} \equiv(\beta \bar{\beta})^{\ell} e^{-Q \ell \phi}, \quad \ell=1,2, \ldots,\left[\frac{k-1}{2}\right]
$$

where

$$
\beta^{\ell}=\left(\partial^{l} e^{-w}\right) e^{w}=-P_{\ell}(\partial w, \cdots) .
$$

Here, $\beta, \bar{\beta}$ are induced from the Wakimoto free field description of the underlaying $\mathrm{SL}(2, \mathbb{R})$ theory.

The explicit form of $P_{\ell}(\partial w, \cdots)$ was found in [15] for $\ell=1,2,3$. In appendix B, we obtain the general expression (3.5). From (3.4), it is clear that when the excitation level, $\ell$, is much smaller than the curvature radius, $\sqrt{k}$, the wave function of the bound states (3.4) is spread over the whole cap of the cigar.

On the other side of the duality, $W_{\ell}$ takes the form (see equation ${ }^{7}(5.17)$ of [15])

$$
W_{\ell} \equiv e^{i \sqrt{\frac{k_{b}}{2}} \tilde{x}-Q\left(\frac{k_{b}}{2}-\ell\right) \phi}, \quad \ell=1,2, \ldots,\left[\frac{k-1}{2}\right] .
$$

These operators amount to a string winding once around $S_{x}^{1}$, with no angular momentum. The consideration of their anti-winding partners, $W_{\ell}^{*}$, is obvious. It is clear from the exponential factor in $\phi$ that these states are highly localized around the tip of the cigar,

\footnotetext{
${ }^{6}$ We set $\alpha^{\prime}=2$ when it is not presented.

${ }^{7}$ Some sign conventions are different here.
} 
when $\ell \ll \sqrt{k}$, namely, when the excitation number $\ell$ is much smaller than the size of the cap, $\sqrt{k}^{8}$

A detailed description of the GFZZ duality in the $N=(2,2) \mathrm{SL}(2, \mathbb{R}) / \mathrm{U}(1) \mathrm{SCFT}$, which is needed for the black fivebranes, is straightforward, though cumbersome, [63], but the significant issues for the physics of the Euclidean black hole in string theory are isomorphic to those in the bosonic coset CFT.

So far, the discussion was at the CFT level. What does this mean in string theory? For $I_{\ell} \sim W_{\ell}$ to correspond to an on-shell state in string theory, we have to have an extra time direction. Then, the statement in string theory is that two states, one that behaves asymptotically like $\exp (-Q \ell \phi)$ and another that behaves like $\exp \left[\left(-\frac{1}{Q}+(\ell-1) Q\right) \phi\right]$, are in fact two components of the same state. This is, therefore, a situation that does not involve BHs, in which stringy information is radically different than in quantum field theory on curved space-time.

The case $\ell=\bar{\ell}=1$ amounts to the FZZ duality between $I$ and $W=W^{+}+W^{-}$, where

$$
I \equiv I_{1} \equiv I_{1,1}=\partial w \bar{\partial} w e^{-Q \phi},
$$

namely, asymptotically on the $\mathbb{R}_{\phi} \times S_{x}^{1}$ cylinder, $I$ behaves like the graviton operator ${ }^{9}$

$$
I \sim \partial x \bar{\partial} x e^{-Q \phi}
$$

and

$$
W^{+} \equiv W_{1} \equiv W_{1,1}=e^{-w}, \quad W^{-} \equiv W_{1}^{*}=e^{-\bar{w}},
$$

namely, asymptotically on the cylinder, $W$ is a sine-Liouville operator,

$$
W \simeq \cos \left(\frac{\beta}{2 \pi \alpha^{\prime}}\left(x_{L}-x_{R}\right)\right) e^{-\frac{1}{Q} \phi},
$$

where we have reinserted $\alpha^{\prime}{ }^{10}$

\subsection{The $k=1$ transition}

Consider the worldsheet Lagrangian on the cylinder, $d s^{2}=d \phi^{2}+d x^{2}$, with a linear dilaton $(2.10),(2.11)$,

$$
\mathcal{L}_{0}=\partial \phi \bar{\partial} \phi+\partial x \bar{\partial} x-Q \hat{R} \phi
$$

where $\phi$ and $x$ are canonically normalized free fields. Both the graviton operator $I$ in (3.8) and the winding string operator $W$ in (3.10) are truly marginal operators in this theory. Hence, adding either of them to $\mathcal{L}_{0}$ must give rise to a two-dimensional CFT which coincides with the linear dilaton one asymptotically. The interaction $\partial x \bar{\partial} x e^{-Q \phi}$ is the leading behavior in $1 / k$ of a two-dimensional sigma-model on the cigar background, (2.8). Consequently, adding it to $\mathcal{L}_{0}$, the CFT must take care of itself to become the $\operatorname{SL}(2, \mathbb{R}) / \mathrm{U}(1)$

\footnotetext{
${ }^{8}$ It was shown, [49, 60-62], that they are localized at a distance of order the string length, $l_{s}$, around the tip of the cigar, for parametrically small curvature, $Q$.

${ }^{9} \mathrm{Up}$ to a $k$-dependent pre-factor and a total derivative.

${ }^{10}$ In the superstring, (3.10) is an $N=2$ Liouville superfield, with $\beta=\frac{2 \pi}{Q} \sqrt{2 \alpha^{\prime}}$, as in (2.9), while in the bosonic string $\beta=\frac{2 \pi}{Q} \sqrt{2\left(1+Q^{2}\right) \alpha^{\prime}}$.
} 
cigar theory. On the other hand, adding $W$ to $\mathcal{L}_{0}$ gives rise to a sine-Liouville CFT. Now, the FZZ duality implies that the latter must take care of itself to become the $\mathrm{SL}(2, \mathbb{R}) / \mathrm{U}(1)$ cigar CFT as well.

All in all, the FZZ duality means that the graviton $I$ and the winding tachyon $W$ must condense simultaneously on the worldsheet, in a correlated way, so that

$$
\mathcal{L}_{\text {int }}=\lambda_{I} I+\lambda_{W} W
$$

with the size of the winding condensate, $\lambda_{W}$, being related to the size of the graviton condensate, $\lambda_{I}$, in an unambiguous way. Indeed, it is known [30] that

$$
\lambda_{W}=\frac{k}{\pi}\left(\pi \lambda_{I} \Delta(1 / k)\right)^{\frac{k}{2}},
$$

where

$$
\Delta(z) \equiv \frac{\Gamma(z)}{\Gamma(1-z)}
$$

The fact that both $\beta$ and $1 / Q$ in $W,(3.10)$, go like $\sqrt{k}$ at large $k$ means that it is nonperturbative in the $\alpha^{\prime}$ expansion. For large $k$, the winding string condensate, $\lambda_{W} W$ in (3.12), (3.10), decreases rapidly (compared to $I$ ) as $\phi \rightarrow \infty$. This is directly related to the fact that the string winding around the $x$ circle is very heavy there. Conversely, as one moves to smaller $\phi$, the wave function (3.10) grows, and eventually, as one approaches the tip, one can no longer think of the condensate as describing a wound string. The way to think about it, instead, is discussed in the next subsection.

As $k$ decreases, the falloff of the condensate (3.10) becomes less and less rapid. For $k \rightarrow 1$, the wave function of the winding string spreads all the way to infinity. ${ }^{11}$ In this regime, the cigar CFT is better described as a theory on $\mathbb{R} \times S^{1}$ with linear dilaton and a sine-Liouville deformation (3.10). This transformation of the sigma model on the cigar at large $k$ to a sigma model on the cylinder with a sine-Liouville deformation at small $k$ is an outcome of the FZZ correspondence. It is an example of a strong-weak coupling duality on the worldsheet. In general, one should think of the theory as containing both deformations, (3.13) (the cigar geometry and the thermal winding tachyon); which one is dominant depends on the value of $k$.

As shown in [14, 15], and partly reviewed above, the FZZ correspondence has other consequences, visible already at large $k$. For example, wave functions of low-energy normalizable states on the cigar, which typically have an extent $\sqrt{k} l_{s}$ and are well described by supergravity, a.k.a. the excitations $I_{\ell, \bar{\ell}}$ of the graviton condensate, have another component, the excitations $W_{\ell, \bar{\ell}}$ of the winding condensate, which is highly localized near the tip at large $k$. If one probes the Euclidean $\mathrm{BH}$ geometry by scattering, low-energy probes see the cigar geometry, as expected, while high-energy probes see instead the sine-Liouville one [15]. In particular, the hard wall associated with the fact that the radial direction on a cigar ends at a particular point (the tip), is replaced for high-energy probes by a soft wall,

\footnotetext{
${ }^{11}$ The vertex operator (3.10) still falls off at $k=1$, but the corresponding wave function, which differs from the vertex operators by a factor of $e^{\Phi} \sim e^{-\frac{Q}{2} \phi}$, does not.
} 
associated with the sine-Liouville potential (3.10), that recedes to more and more negative $\phi$ as the energy of the probe increases.

Thus, non-perturbative $\alpha^{\prime}$ effects, in particular, the condensation of the closed string tachyon winding around the Euclidean time direction, lead to a significant modification of the physics of Euclidean BHs in classical string theory. These effects are expected to be universal, since Euclidean BHs always contain as part of the geometry a cigar in the radial and Euclidean time direction, and it is natural to conjecture that a string wrapped around the angular direction of that cigar has a non-zero condensate in general $\mathrm{BH}$ backgrounds. In particular, we regard the transition at $k=1$, a.k.a. when the size of the $\mathrm{BH}$ is the string length scale, $l_{s}$, as a (post-)hint to a $\mathrm{BH}$-string transition in string theory: the $\mathrm{BH}$ states in string theory on flat space-time with a linear dilaton cease to exist when $k \leq 1$, and the density of high-energy states is dominated entirely by perturbative string states.

It was checked [48] that indeed the Bekenstein-Hawking entropy is bigger than that of perturbative string states when $k>1$, it precisely matches the one of perturbative string states in an asymptotically flat space-time with a linear dilaton when $k=1$, while the latter is bigger than the former when $k<1$. For other BHs in string theory, e.g. the fourdimensional Schwarzschild BH, a similar transition is conjectured, [46, 47, 49], when the Schwarzschild radius is of the order of $l_{s}$. Although the details of the transition are different - in the $2 d$ Schwarzschild-like case the order parameter is $k$ while in the $4 d$ Schwarzschild case it is the string coupling $g_{s}$, the nature of the transition is similar: in both cases the transition occurs when the curvature length of the cap of the Euclidean BH's cigar is $l_{s}$.

The main lesson that we take from the above is the following. Having an exact worldsheet CFT for the two-dimensional $\mathrm{BH}$, which thus allows us to inspect precisely the nonperturbative effects hinting a $\mathrm{BH}$-string transition, a.k.a. the winding string condensate, leads to a (post-)suggestion for similar properties regarding the physics of general BHs in string theory. Another non-perturbative hint from the Euclidean cigar CFT, to the nature of the BH-string transition at $k=1$, will appear in the next subsection.

\subsection{The fusion $\mathrm{F} \sim \mathrm{W}^{+} * \mathrm{~W}^{-}$}

In this subsection, we discuss a novel duality that, motivated by the results of $[28,30]$, was proposed recently in [24]. This duality is key for the understanding of non-perturbative $\alpha^{\prime}$ effects in the black fivebranes.

The duality is between the cigar CFT described by the FZZ condensation (3.12) in a linear dilaton theory (3.11), and the one obtained by a different condensate,

$$
\mathcal{L}_{\text {int }}=\lambda_{I} I+\lambda_{F} F,
$$

where $F$ is the fusion of $W^{+}$and $W^{-}$(defined in (3.9) with (3.3)). The concrete meaning of the fusion is

$$
F(w) \sim \int d^{2} z W^{+}(z) W^{-}(w) .
$$

The normalization is such that, for integer $k$,

$$
F=(\beta \bar{\beta})^{k} e^{-\frac{2}{Q} \phi},
$$


where $\beta^{k}$ is given in terms of $\phi$ and $x$ in (3.5) and

$$
\lambda_{F}=\frac{\left(\pi \lambda_{I} \Delta(1 / k)\right)^{k}}{\pi \Delta(k)},
$$

where $\Delta(z)$ is given in (3.14).

Note that $F$ is formally the same as $I_{k}$ in (3.4), but it is not an excitation of a graviton. Since $k>\left[\frac{k-1}{2}\right]$, it is outside the unitarity bound and it does not generate a state in the theory. More precisely, it does not generate a single string state in the theory. Indeed, (3.16) implies that it should be viewed as a bound state of $W^{+}$and $W^{-}$, which are states in the theory. Moreover, $k$ need not be an integer and so generically (3.17) is not well defined. We shall get back to this important point.

The precise meaning of this conjectured duality is discussed in [24]. In short, one may calculate correlators using either (3.12) or (3.15). As long as the size of the condensate of $F$ is related to that of $W$ by (3.18) combined with (3.13), one obtains the same result. Concretely, inside correlation functions, ${ }^{12}$

$$
\left(\lambda_{W}\right)^{2} \int d^{2} z W^{+}(z) W^{-}(w)=C_{W F} \lambda_{F} F(w),
$$

with

$$
\left(\lambda_{W}\right)^{2}=C_{W F} \lambda_{F},
$$

that is compatible with (3.15), (3.18) and (3.12), (3.13), a.k.a.

$$
C_{W F}=-\pi \Delta(-k)\left(\lambda_{W}\right)^{2},
$$

which was verified by a direct calculation in [24]. For the two and three-point functions, this $F-W$ conjecture was checked by combining the results of [30] and [28].

Our main physics motivation for such a duality is that it provides a sensible way to think about the winding condensate of the previous subsection near the tip. For a large Euclidean BH, the condensate $F$ in (3.16) (with (3.5) and (3.3) inserted) behaves like

$$
F \sim(\partial(\phi-i x) \bar{\partial}(\phi+i x))^{k} e^{-\sqrt{2 k} \phi}+\ldots,
$$

where the '... ' stand for corrections in $1 / k$. It thus describes a highly excited string that is highly localized near the tip of the cigar. It is reasonable to think about the winding string condensate in this way: near the Euclidean horizon, the winding one string condensate, $W^{+}$, tends to form a bound state with its conjugate, $W^{-}$, giving rise to a highly excited string condensate, instead.

For a small Euclidean BH, the description of the cigar CFT in terms of (3.15) adds another ingredient to the BH-string transition of the previous subsection. When $k=1$, the graviton operator $I$ and the winding-anti-winding bound-state operator $F$ have the following properties. First, as can be seen from (3.7) with (3.5) versus (3.17),

$$
F=I=\beta \bar{\beta} e^{-\sqrt{2} \phi} \quad \text { when } \quad k=1 .
$$

\footnotetext{
${ }^{12}$ In winding non-conserving correlators, one uses the winding one, $h=\bar{h}=0$ degenerate operator, as was proposed in [21]; for a review, see e.g. subsection 2.6 in [58].
} 
Second, the operator in (3.23) is FZZ dual to a winding operator $W$ whose wave function is marginally non-normalizable $(I, W$ and $F$ are at the unitarity bound in the $\mathrm{SL}(2, \mathbb{R}) / \mathrm{U}(1)$ CFT when $k=1$; see appendix E). Moreover, when $k<1$, the winding-anti-winding fused operator $F$ generates a normalizable state. On the other hand, even though the wave function of the graviton $I$ is normalizable, the wave function of its FZZ dual $W$ is not normalizable when $k<1$ ( $I$ and $W$ are outside the unitarity bound in this case; see appendix E). This means that below the $k=1$ transition point, the description of the worldsheet CFT in terms of the geometry of a cigar is misleading. Instead, the theory is described in terms of perturbative fundamental strings, which amount to the condensate $F \sim W * W^{*}$.

All in all, the FZZ duality together with the fusion, $F \sim W * W^{*}$, leading to the $F-W$ duality conjecture above, imply that the tip of the cigar is special already in classical string theory, due to stringy physics that is non-perturbative in $\alpha^{\prime}$, and which can neither be detected via GR considerations, nor taking into account perturbative corrections in $\alpha^{\prime}$. We regard it as a Euclidean hint to the possibility that the physics of $\mathrm{BH}$ horizons and/or their interiors is modified dramatically already at the classical level in string theory. Concretely, it hints to the possibility that the $\mathrm{BH}$ interior is a string condensate, leading to the following.

For small BHs, it hints to a $\mathrm{BH}$-string transition, as the size of the $\mathrm{BH}$ approaches $l_{s}$, and when the perturbative string condensate is being 'released' from its interior. For large $\mathrm{BHs}$, we regard the non-perturbative properties of classical string theory on the cigar CFT as a hint to a realization of a firewall or a fuzzball. This will be discussed in section 4, but prior to turning to the Lorentzian case, we continue to collect more Euclidean hints.

\subsection{The fusion $F_{\ell} \sim W * W_{\ell}^{*}$}

In subsection 3.1, we reviewed the GFZZ duals of the GR-like excitations $I_{\ell, \bar{\ell}}$ - the winding one stringy modes $W_{\ell, \bar{\ell}}$ - in the cigar CFT. In string theory on the Euclidean black hole (times an extra real time), while the GR modes are spread in the cigar cap, their stringy non-perturbative dual partners are localized at the tip. This suggests [15] that, at least naively, in string theory the information in the $\mathrm{BH}$ atmosphere is stored also at the $\mathrm{BH}$ horizon (and possibly also in the BH interior, that is absent in the Euclidean geometry). This exciting possibility will be confirmed in the next sections.

Recall, though, that our motivation for considering string theory on the Euclidean black hole (times an extra real time), is to collect useful hints regarding the physics of string theory on the Lorentzian $\mathrm{BH}$, in a simple, well understood setup. As we shall discuss in section 4, non-perturbative stringy aspects of perturbative string theory on the Lorentzian $\mathrm{BH}$ have challenging subtleties, yet to be understood. Those are ameliorated, once we describe the theory in its dual description (3.15). In the latter framework, the non-perturbative stringy partners of $I_{\ell, \bar{\ell}}$, which we denote by $F_{\ell, \bar{\ell}}$, are obtained by the fusion of $W_{\ell, \bar{\ell}}$ with $W$. So, next, we describe these excitations of $F$, in string theory on the Euclidean BH. 
Again, for simplicity we take $\ell=\bar{\ell}$, and consider the fusion of the winding string condensate $W^{+},(3.9)$, with the excited winding operators $W_{\ell}$ in $(3.6),{ }^{13}$

$$
F_{\ell}(w) \sim \int d^{2} z W^{+}(z) W_{\ell}^{*}(w), \quad \ell=1,2, \ldots,\left[\frac{k-1}{2}\right] .
$$

The normalization is such that, for integer $k$,

$$
F_{\ell}=(\beta \bar{\beta})^{k+1-\ell} e^{-Q(k+1-\ell) \phi},
$$

where $\beta^{k+1-\ell}$ is defined in eq. (3.5) with (3.3).

The calculation leading to (3.24), (3.25) is similar to the one done in the particular $\ell=1$ case in [24], mentioned in the previous subsection, and the meaning of (3.25) for generic $k$ is also similar to that of its low-lying $F=F_{1}$ condensate, (3.16), (3.17), as we discuss next.

The $F_{\ell}$ in (3.24), (3.25) are equal formally to the expressions $I_{k+1-\ell}$ in (3.4), but, just like $F$, they are not excitations of the gravity states that are generated by the operators $I_{\ell}$ in (3.4). The reason is the following. Recall, [15], that the range of the excitation number $\ell$ for the GR bound states in the cap is $\ell=1,2, \ldots,\left[\frac{k-1}{2}\right]$. In the worldsheet CFT on the cigar, the bound $\ell \leq\left[\frac{k-1}{2}\right]$ in (3.4) follows from the unitarity bound for such states in affine $\operatorname{SL}(2, \mathbb{R})_{k}$. So, actually, the operators $F_{\ell}$ in (3.24) do not generate states in the theory, since $k+1-\ell>\left[\frac{k-1}{2}\right]$, and thus they are outside the unitarity bound.

Again, just like the ground-state $F$, its excitations $F_{\ell}$ do not correspond to single string states, but to a bound state of $W$ and $W_{\ell}$. Moreover, generically, $k+1-\ell$ is not an integer, in which case (3.25) is a notation for its definition in eq. (3.24). Indeed, for generic $k$, the $F_{\ell}$ in (3.24) can be thought of as a smeared average over off-shell string excitations, which collapses to an on-shell excitation for integer $k$. Note that this is in harmony with the analytic structure of the condensate size, $\lambda_{F}$, in eqs. (3.15)-(3.19): one may regard the function $\lambda_{F}(k),(3.18)$, as a measure of the smearing, in particular, it develops a pole when $k$ is integer, in harmony with the collapse of the condensate $F$ and its excitations $F_{\ell}$ to on-shell operators, (3.25), in that case.

To recapitulate, the operators $F_{\ell}$ in eqs. (3.24), (3.25) are the non-perturbative partners of $I_{\ell}$. They describe excited modes of the fused condensate, $F \sim W * W^{*}$, and are highly localized near the tip of the cigar (as long as the excitation number, $\ell$, is sufficiently smaller than the size of the cap, $\sqrt{k}$ ).

The scaling dimension of $F_{\ell}$ is identical, by construction, (3.24), to that of $W_{\ell}$ in (3.6) and thus also to $I_{\ell}$ in (3.4),

$$
\Delta(\ell)=\ell-\frac{\ell(\ell-1)}{k},
$$

as it must. Note that $\Delta(\ell) \geq 1$, and $\Delta=1$ only for $\ell=1$. Therefore, when considering string theory on the cigar, $I_{\ell}, W_{\ell}$ and $F_{\ell}$, with $\ell>1$, can appear as on-shell physical states only if we have an extra time direction.

Nevertheless, we regard the duality between the excitations of the fused condensate near the tip and the gravity bound states in the cap as a Euclidean hint to a duality

\footnotetext{
${ }^{13}$ The fusion $F_{\ell, \bar{\ell}}$ of $W^{+}$with generic $W_{\ell, \bar{\ell}}$ is obvious; it is presented in appendix A.
} 
between the modes in the interior of the $\mathrm{BH}$ and those in its thermal atmosphere. This will be clarified in sections 4 and 5 , but prior to turning to the Lorentzian case, we present another intriguing Euclidean hint in the next subsection.

\section{5 $F=I^{k}$ and $\mathrm{BH}=\mathrm{GC}$}

In this subsection, we shall argue that, in a certain sense, $F$ may be regarded also as a bound state of $k$ gravitons, localized near the tip of the cigar. Concretely, for integer $k$, the operator $F$ in (3.19) takes the form (3.17), which can be written schematically as

$$
F=\left(\beta \bar{\beta} e^{-Q \phi}\right)^{k}=I^{k}
$$

this follows from (3.17) versus (3.7) with (3.5).

More precisely, it was argued in [28] that, inside correlators,

$$
\left(\lambda_{I}\right)^{k}\left[\prod_{i=1}^{k-1} \int d^{2} z_{i} I\left(z_{i}\right)\right] I(w)=C_{I F} \lambda_{F} F(w)
$$

with

$$
\left(\lambda_{I}\right)^{k}=C_{I F} \lambda_{F}
$$

for the $\lambda_{F}$ in (3.15)-(3.18).

There is a problem with eq. (3.28): the entire discussion is valid for integer $k$, but for such $k$ 's $\lambda_{F}(k)$ diverges. ${ }^{14}$ Still, with manipulations that involve, in particular, continuation in $k$, in [28], it was shown that the two and three point functions calculated with the screening operator $F$ are identical to those calculated with the screening operator $I$, if (3.28) is satisfied with (3.29), (3.18).

Despite the fact that we do not have a physical understanding of these manipulations and the fact that, unlike $F \sim W * W^{*},(3.28)$ is defined only for integer $k$, we find it intriguing, especially since this seems to resonate with ideas presented in [44, 45]. There, it was argued that $\mathrm{BHs}$ are filled with a condensate of gravitons. In the next section, we argue that $F$ is the operator that corresponds to a BH-filling folded string. Consequently, the relation in eq. (3.28) implies that, at least far from the $\mathrm{BH}$, the folded string might be viewed as a bound state of $k$ gravitons.

\section{Lorentzian physics}

In this and the next sections, we consider the Lorentzian black fivebranes, and see how the Euclidean hints from the previous section turn into rather concrete statements, regarding what we refer to as stringy information.

In the first subsection, we present the subtleties associated with the continuation of $W$ to the Lorentzian case. In the second subsection, we turn to the physics of $F$, which avoids these subtleties, and argue that it amounts to an interior-filling folded string. Finally, in the last subsection, we discuss the light it sheds on the BH-string transition.

\footnotetext{
${ }^{14} \mathrm{~A}$ possible interpretation of this divergence is discussed in the previous subsection.
} 
In particular, we argue in this section that $F$ entangles the two sides of the eternal $\mathrm{BH}$, similar to $\mathrm{ER}=\mathrm{EPR}$, whose precise realization as stringy information is clarified when considering the excitations of the stringy $\mathrm{BH}$, in the next section.

\subsection{I and $W$}

As discussed in section 2 , the Lorentzian $\mathrm{SL}(2, \mathbb{R})_{k} / \mathrm{U}(1)$ geometry can be obtained e.g. by the analytic continuation

$$
i x \rightarrow t .
$$

In particular, after analytic continuation, the graviton operator (3.8) becomes

$$
I \sim \partial t \bar{\partial} t e^{-Q \phi},
$$

up to a total derivative. This is in harmony with the behavior of the two-dimensional BH geometry in (2.6), at asymptotically large radial $\phi$. And, as in the Euclidean case, once $I$ is condensed in the $\mathbb{R}_{\phi} \times \mathbb{R}_{t}$ theory with a linear dilaton - the continuation of (3.11), the theory 'takes care' of itself and becomes the Lorentzian $\mathrm{SL}(2, \mathbb{R})_{k} / \mathrm{U}(1)$ quotient CFT.

The coordinates $-\infty \leq t \leq \infty$ and $\phi \geq 0$ cover the right wedge of an eternal $\mathrm{BH}$; the former can be extended to the latter geometry in the standard way, e.g. by using Kruskal-Szekeres coordinates, $-\infty \leq u, v \leq \infty$ (see e.g. [53], for a review),

$$
v=\sinh \left(\frac{2 \pi \phi}{\beta}\right) e^{2 \pi t / \beta}, \quad u=-\sinh \left(\frac{2 \pi \phi}{\beta}\right) e^{-2 \pi t / \beta},
$$

in terms of which the two-dimensional BH metric and dilaton in (2.6) and (2.7) take the form

respectively. ${ }^{15}$

$$
d s^{2}=-\frac{d u d v}{1-u v}, \quad e^{2 \Phi}=\frac{g_{0}^{2}}{1-u v},
$$

The $\beta$ in (4.3) is the inverse Hawking temperature of the BH. Recall that in the bosonic $\mathrm{SL}(2, \mathbb{R})_{k_{b}} / \mathrm{U}(1)$ and fermionic $\mathrm{SL}(2, \mathbb{R})_{k} / \mathrm{U}(1)$ theories, $\beta$ is related to the bosonic level, $k_{b}=k+2$, and the total one, $k$, by

$$
\beta=2 \pi \sqrt{\alpha^{\prime} k_{b}} \quad \text { (bosonic); } \quad \beta=2 \pi \sqrt{\alpha^{\prime} k} \quad \text { (fermionic), }
$$

respectively (recall also that we set $\alpha^{\prime}=2$, when it is not presented explicitly).

The analytic continuation (4.1) of the sine-Liouville operator (3.10) gives ${ }^{16}$

$$
W \rightarrow \cosh \left(\sqrt{\frac{k_{b}}{2}}\left(t_{L}-t_{R}\right)\right) e^{-\frac{1}{Q} \phi} .
$$

\footnotetext{
${ }^{15}$ The idea that the condensation of $I$ forms the ER bridge is the following. One begins with the theory (3.11) with (4.1). This theory is strongly coupled at negative $\phi$, and thus it is not well defined there. Then, upon condensing the operator $I$ in (4.2), the theory 'takes care' of itself and becomes the Lorentzian $\mathrm{SL}(2, \mathbb{R})_{k} / \mathrm{U}(1)$ quotient $\mathrm{CFT}$, as discussed below eq. (4.2). From the structure of the underlying $\mathrm{SL}(2, \mathbb{R})$, we know that the geometry includes the whole $-\infty<u, v<\infty$ space-time in eq. (4.4), a.k.a the eternal black hole. This is the sense by which the condensation of $I$ forms an ER bridge, as discussed in the introduction.

${ }^{16}$ As in section 3 , we present the bosonic case; similar manipulations can be done straightforwardly in the fermionic case of our ultimate interest, giving rise to the same result.
} 
Since $W$ arises from an $\mathrm{SL}(2, \mathbb{R})_{L} \times \mathrm{SL}(2, \mathbb{R})_{R}$ invariant operator in the underlying $\mathrm{SL}(2, \mathbb{R})$ theory, it must survive any gauging, in particular, the one leading to the Lorentzian $\mathrm{SL}(2, \mathbb{R}) / \mathrm{U}(1) \mathrm{CFT}$. This, however, seems to lead to some non-conventional, apparently inconsistent properties.

For instance, consider the following operator product expansion (OPE):

$$
e^{i E t(z, \bar{z})} e^{\sqrt{k_{b} / 2}\left(t_{L}-t_{R}\right)} \sim e^{\vartheta \sqrt{2 k_{b}} E} e^{i\left(E-i \sqrt{k_{b} / 2}\right) t_{L}+i\left(E+i \sqrt{k_{b} / 2}\right) t_{R}},
$$

where here $t(z, \bar{z})=t_{L}(z)+t_{R}(\bar{z}), t_{L}=t_{L}(0), t_{R}=t_{R}(0)$, and $z=|z| e^{i \vartheta}$. The expression (4.7) appears within OPEs of operators corresponding to generic energy states $V_{E}$ in the BH CFT with the screening operator $W$ in (4.6). This leads to non-locality on the worldsheet, since upon rotating $z$ around the origin, $\vartheta \rightarrow \vartheta+2 \pi$, the r.h.s. of (4.7) picks up a factor of $e^{\beta E}$, with the $\beta$ in eq. (4.5).

We should note, however, that we are considering an embedding of a Euclidean worldsheet in a non-trivial Lorentzian space-time. So, it is possible that the non-locality puzzle above is resolved when one considers Lorentzian worldsheets in the eternal $\mathrm{BH}$ target. However, at the moment, it is not known to us how to do it directly. Nevertheless, for Euclidean worldsheets in the Lorentzian BH background, we suggest that this should be resolved in the following way. Going around an insertion of (4.6) on the worldsheet, amounts in space-time to taking

$$
t \rightarrow t+i \beta
$$

The eternal BH coordinates, $u$ and $v$ in (4.3), are invariant under (4.8), and since (4.7) is invariant when combining (4.8) with

$$
\vartheta \rightarrow \vartheta+2 \pi
$$

it is natural to suspect that the apparent non-locality on the worldsheet is not a 'bug,' but rather a 'feature' of the eternal BH theory, which reflects the fact that the $\mathrm{BH}$ has a temperature.

In appendix C, we show how (4.8) and (4.9) come about when considering the Lorentzian $A d S_{3}$ with a Euclidean worldsheet. Since the Lorentzian $A d S_{3}$ CFT is expected to be a consistent target space for perturbative strings, this is a strong support that the issue is with attempting to describe the physics of strings in a non-trivial Lorentzian background using a Euclidean worldsheet.

Luckily, as reviewed in the previous section, in order to describe the non-perturbative $\alpha^{\prime}$ corrections in the $\mathrm{BH}$ case, we do not have to deal with these issues. Instead, we can use the dual description in which $W^{ \pm}$do not condense, but $F$ does. Since $F$ is mutually local with $V_{E}$, this dual description appears to be much simpler. And so next, we turn to discuss the physics of $F$ in the eternal BH theory.

\section{2 $F$, folded string and EPR}

The analytic continuation of $F$ is the fusion, (3.16), of the analytic continuation of $W^{ \pm}$,

$$
W^{ \pm}=\exp \left( \pm \frac{\beta}{2 \pi \alpha^{\prime}}\left(t_{L}-t_{R}\right)\right) e^{-\frac{1}{Q} \phi}
$$


where $\beta$ is given in (4.5). As is clear from (3.16) and (4.10), $F$ is mutually local with respect to $V_{E}$, and thus there are no subtleties associated with its condensation. This implies that $F$ should have a clear interpretation in the $\mathrm{BH}$ geometry.

The goal in this subsection is to discuss the physical meaning of $F$. We claim that the semi-classical meaning of $F$ is of a folded string that is filling the entire $\mathrm{BH}$.

The first evidence for this comes from the target-space interpretation of $F \sim W^{+} * W^{-}$, as presented in figure 1. There, we see how the Hartle-Hawking wave-function procedure fits neatly with $F \sim W^{+} * W^{-}$, provided that $F$ is associated with a folded string that folds towards the $\mathrm{BH}$, as discussed in [32]. Classically, the folded string can fold towards the $\mathrm{BH}$ only behind the horizon $[32,64]$. Outside the $\mathrm{BH}$, the probability for the folded string to fold towards the $\mathrm{BH}$ is exponentially suppressed, as reflected by the $\phi$ dependence of $W^{ \pm}$and $F$, [24]. The fact that $W^{ \pm}$and $F$ are related via analytic continuation can also be seen at the classical level, as illustrated in appendix $\mathrm{C}$.

The argument presented above is valid for any $k$. In the special cases of integer $k$, another, more direct, argument can be made. The asymptotic behavior of the operator $F$ in the Lorentzian BH is obtained by the analytic continuation, (4.1), of eqs. (3.17), (1.1), giving rise to

$$
F \sim(\partial(\phi-t) \bar{\partial}(\phi+t))^{k} e^{-\frac{2}{Q} \phi}+\ldots
$$

where the '...' stand for corrections in $1 / k$, which can be read from the analytic continuation (4.1) of (3.17) with (3.5) and (3.3). Equation (4.11) implies that far from the BH, $F$ describes a level $k$ on-shell excitation of a string whose size is $\sqrt{k}$. Now, since the size of the $\mathrm{BH}$ is also $\sqrt{k}$ and since (4.11) implies that the wave function of the string is highly suppressed away from the $\mathrm{BH}$, it is natural to interpret $F$ as a folded string that semiclassically is filling the $\mathrm{BH}$ interior. This is also reflected in $\lambda_{F}(k)$, which develops a pole for integer $k$. For non-integer $k,(4.11)$ does not hold, and $\lambda_{F}(k)$ is regular. Since (3.24) is still valid, we expect the size of the string to be $\sqrt{k}$ in average.

A third evidence comes from the fact that $F$ is a screening operator, a.k.a. it is reduced from a truly marginal operator with $J^{a}=\bar{J}^{a}=0$ in the underlying $\operatorname{SL}(2, \mathbb{R})$ theory. This agrees with the observation made in $[32,33]$ that the $\mathrm{BH}$-filling folded string does not break any of the $\mathrm{BH}$ background symmetries.

In light of these arguments, we assume in the rest of the paper that $F$ is the operator that corresponds to the folded strings discussed in [32, 33], and see what physics follows from this assumption.

One conclusion is that the black fivebranes system realizes the ER=EPR proposal, [6], albeit with a twist. To see this, we recall that $I$ is the operator that creates the ER bridge between the two asymptotic regions. As we just argued, $F$ corresponds to a folded string. This folded string is an extended object with tails on both asymptotic regions, and so it entangles the right and the left sides of the $\mathrm{BH}$. It is natural, therefore, to relate $F$ with the EPR side of the duality. The fact that the folded string entangles the left wedge with the right wedge is made more precise in the next section. There, we consider excitations of the eternal $\mathrm{BH}$. We show that an excitation that lives on, say, the right wedge of the $\mathrm{BH}$, 
is accompanied with an excitation of the folded string with tails both on the right and the left wedges.

The twist is that the CFT statement is not that $I=F$ (which is the analog of $\mathrm{ER}=\mathrm{EPR}$ ). Operationally, such a statement would mean that one can either work with the screening operator $I$ or with the screening operator $F$. As discussed above, the correct statements is that both $I$ and $F$ must condense. ${ }^{17}$ Namely, the condensate is of $\lambda_{I} I+\lambda_{F} F$. In other words, the ER bridge is not empty, but is filled with folded strings.

Before considering, in the next section, excitations of the $\mathrm{BH}$ and folded string, which strengthen the above picture about stringy information, we first comment about the BHstring transition [46, 47] for black fivebranes [48, 49], in the next subsection.

\section{3 $k=1$ and the BH-string transition}

The semiclassical description of the condensate $F$ in terms of an interior-filling folded string sheds new light on the nature of the transition at $k=1$, discussed in the Euclidean case in subsections 3.2 and 3.3. In particular, it clarifies its interpretation as realizing the BH-string transition, for the $\mathrm{SL}(2, \mathbb{R})_{k} / \mathrm{U}(1)$ Lorentzian BH. Next, we discuss it in some detail.

For parametrically large $k$, the folded string is trapped in the interior of the $\mathrm{BH}$, with a tail, $W$, highly localized at a distance $l_{s}$ from the horizon. The geometric description of the $\mathrm{BH}$ interior is thus misleading; an infalling observer will encounter the strings condensate as s.he approaches a distance $l_{s}$ from the horizon. Even as $k$ decreases, as long as $k$ is large, the change in the effect of the strings condensate outside the $\mathrm{BH}$ is hardly felt. However, as $k \rightarrow 1$, the effect of the folded string changes dramatically. The range of its tail is expanded in the radial direction all the way to $\phi \rightarrow \infty$.

For $k<1$, two things happen at once. On the one hand, as in the Euclidean case, the graviton condensate, $I$, which behaves asymptotically like $e^{-Q \phi}$, has $j+1=1$, and is thus outside the unitarity bound: $j=0>\frac{k-1}{2}$ (see appendix E). Consequently, the description of the $\mathrm{SL}(2, \mathbb{R})_{k} / \mathrm{U}(1)$ theory in terms of the geometry of a $\mathrm{BH}$, as in eq. (2.6) and/or eq. (4.4), is misleading - the BH is not a state in the $\mathbb{R}_{t} \times \mathbb{R}_{\phi}$ theory with a linear dilaton. On the other hand, the fundamental strings condensate, $F$, which behaves asymptotically like $e^{-Q k \phi}$, has $j+1=k$ (see appendix E), and is thus inside the unitarity bound when $1 / 2<k<1:-\frac{1}{2}<j=k-1<\frac{k-1}{2}$. Note that the lower bound on $k$ follows from the fact that in the superstring, the central charge of the $\mathrm{SL}(2, \mathbb{R})_{k} / \mathrm{U}(1)$ worldsheet SCFT is $c=3+\frac{6}{k}$, and thus it is over critical, $c>15$, when $k<1 / 2$.

The transition point, $k=1$, is very special. At this point, both the fundamental strings screening operator and the gravitons screening operator are at the boundary of the unitarity bound; they both have $j=0=\frac{k-1}{2}$. And, actually, as in the Euclidean case, (3.23), at this point, the operators $F$ in (4.11) and $I$ in (4.2) coincide,

$$
F=I \sim \partial t \bar{\partial} t e^{-\sqrt{2} \phi}
$$

up to a total derivative.

\footnotetext{
${ }^{17}$ For example, $I$ and $F$ are responsible for different poles in correlation functions [43].
} 
The picture that emerges from the above is thus the following. At the transition point, $k=1$, the localized folded strings, which, in a sense, replace the $\mathrm{BH}$ interior, are being 'released,' as perturbative strings. To recapitulate, while for $k>1$, the better description of the $\mathrm{SL}(2, \mathbb{R})_{k} / \mathrm{U}(1)$ Lorentzian theory is in terms of a $\mathrm{BH}$ background, whose interior is being replaced by folded strings, for $k<1$, the $\mathrm{BH}$ disappears, but the strings remain. This provides a nice realization of the $\mathrm{BH}$-string transition, in the two-dimensional case.

\section{$5 \quad \mathrm{BH}$ and stringy information}

In this section, we discuss excitations of the eternal BH. We show that excitations of ordinary GR modes that are confined to the $\mathrm{BH}$ atmosphere are accompanied with excited folded string modes that live mostly inside the BH. We discuss the implications of this observation.

Let us start by reviewing some well known facts [11] about ordinary GR-like modes that propagate in the $\mathrm{SL}(2, \mathbb{R})_{k} / \mathrm{U}(1) \mathrm{BH}$ atmosphere. Consider a massless minimally coupled scalar field, $a$, that propagates in the eternal BH geometry that takes the form, (2.6), (2.7),

$$
d s^{2}=-\tanh ^{2}(\phi / \sqrt{2 k}) d t^{2}+d \phi^{2}, \quad e^{2 \Phi}=g_{0}^{2} \cosh ^{-2}(\phi / \sqrt{2 k}) .
$$

Defining $\Psi(\phi)=a(\rho) \cosh (\phi / \sqrt{2 k}) e^{-i E t}$ and performing the coordinate transformation $\rho=$ $\sqrt{2 k} \log (\sinh (\phi \sqrt{2 k}))$, we obtain a Schrodinger-like equation in the tortoise coordinates, $\rho$,

$$
\left(-\partial_{\rho}^{2}+V(\rho)\right) \Psi(\rho)=E^{2} \Psi(\rho),
$$

with

$$
V(\rho)=\frac{1}{2 k}\left(1-\frac{1}{(1+\exp (2 \rho / \sqrt{k}))^{2}}\right) .
$$

Note that, much like in Schwarzschild BH, the size and hight of $V(\rho)$ are fixed by the curvature, $1 / k$ in our case. However, here, unlike in Schwarzschild BH, due to the linear dilaton, $V(\rho)$ is a monotonic function. ${ }^{18}$

This implies that there are two kinds of modes (see figure 2). Modes with $E^{2}>1 / 2 k$ oscillate both at infinity, $\rho \rightarrow \infty$, and at the horizon, $\rho \rightarrow-\infty$. We refer to such modes as scattering modes. There are also bound state modes, with $1 / 2 k>E^{2}>0$; these modes are oscillating at the horizon and decay exponentially fast at infinity, and so they are indeed bounded to the BH. ${ }^{19}$ From the underlying $\mathrm{SL}(2, \mathbb{R})$ point of view, the scattering states arise from the principal continuous representations, while the bound states arise from the principal discrete representations. To see this, we recall how these, and other modes that propagate in the $\mathrm{SL}(2, \mathbb{R}) / \mathrm{U}(1) \mathrm{BH}$ geometry, are described in string theory.

Consider, for example, type II string theory on $\mathrm{SL}(2, \mathbb{R})_{k} / \mathrm{U}(1) \times \mathcal{N}$, namely, on the two-dimensional BH times an 'internal space' $N=2 \mathrm{SCFT}, \mathcal{N}$. For instance, in the theory of $k$ near-extremal NS fivebranes on a five torus, discussed in section $2, \mathcal{N}=\mathrm{SU}(2)_{k} \times T^{5}$

\footnotetext{
${ }^{18}$ This is a consequence of considering the near fivebrane theory, (2.6); in the full background of the black fivebrane, (2.1), $V(r)$ is a step function, as in Schwarzschild BH.

${ }^{19}$ In Schwarzschild BH, the situation is similar; the only difference is that the modes in the atmosphere can tunnel to infinity, as the potential goes to zero there.
} 
(or $\mathbb{R}^{5}$, in the special case of a non-compact black fivebrane). A class of physical vertex operators of interest, in the $(-1,-1)$ picture, are (see e.g. [65], for a review and definitions)

$$
V=e^{-\varphi-\bar{\varphi}} V_{j E} V_{\mathcal{N}},
$$

where $V_{j E}$ is a primary operator in the $\mathrm{SL}(2, \mathbb{R})_{k} / \mathrm{U}(1) \mathrm{SCFT}$, whose scaling dimension is

$$
h\left(V_{j E}\right)=-\frac{j(j+1)}{k}-\frac{E^{2}}{2},
$$

with the energy, $E$, related to the eigenvalue $m_{2}$ of $J^{2}$ in the underlying $\operatorname{SL}(2, \mathbb{R})_{k}$ theory by

$$
E=Q m_{2},
$$

and similarly for $\bar{h}$ (see e.g. [11, 53], for a review). The on-shell condition reads:

$$
-\frac{j(j+1)}{k}-\frac{E^{2}}{2}+N-\frac{1}{2}=0,
$$

where $N$ is the left-handed dimension of $V_{\mathcal{N}}$, which corresponds to a transverse lefthanded string excitation in $\mathcal{N}$. A similar condition is obtained for the right-moving excitations, $\bar{N}(=N)$.

The modes that satisfy (5.2) correspond to $N=\bar{N}=1 / 2$. There are two ways to satisfy (5.7) with $N=1 / 2$. Modes with $E^{2}>1 / 2 k$ have

$$
j=-\frac{1}{2}+i s,
$$

where $s$ is related to the momentum in the radial direction, $P$, by

$$
P=Q s
$$

These are the scattering modes, which according to (5.8) are reduced from the principal continuous representations (see figure 2).

There are also modes below the mass gap, with $E^{2}<1 / 2 k$. Equation (5.7) with $N=1 / 2$ implies that such modes have real $j$. Namely, they are reduced from the principal discrete representations (for a review on $\mathrm{SL}(2, \mathbb{R})$ representations, see e.g. [66]). States $V,(5.4)$, that are reduced from the principal discrete representations of $\mathrm{SL}(2, \mathbb{R})$ are accompanied with a GFZZ dual, that we denote by $W_{V}$.

To construct $W_{V}$, we first recall that the operators $V_{j E}$ are reduced via coset decomposition from $J^{2}$ (and $\bar{J}^{2}$ ) eigenstates in the underlying theory, hence, we work in the hyperbolic basis of $\mathrm{SL}(2, \mathbb{R})$, and denote such states by $V_{j ; m_{2}}$, where $m_{2}$ is the value of $J^{2}$ (and $\bar{m}_{2}=m_{2}$ below), which is related to the energy $E$ by eq. (5.6). Each $V_{j ; m_{2}}$ can be written as an infinite sum over states in the standard basis, $V_{j ; m_{3}}$, where $m_{3}$ is the value of $J^{3}$.

Now, each $V_{j ; m_{3}}$ has a known GFZZ dual and, consequently, so is each $V_{j ; m_{2}}$, whose dual we shall denote by $\Phi_{\tilde{j} ; m_{2}}^{\omega=1}$. Reducing the latter to the $\mathrm{SL}(2, \mathbb{R}) / \mathrm{U}(1)$ coset, will give rise to the GFZZ duals of GR bound states $V_{j E}$, which we denote by $V_{j E}^{\omega=1}$. Together with 


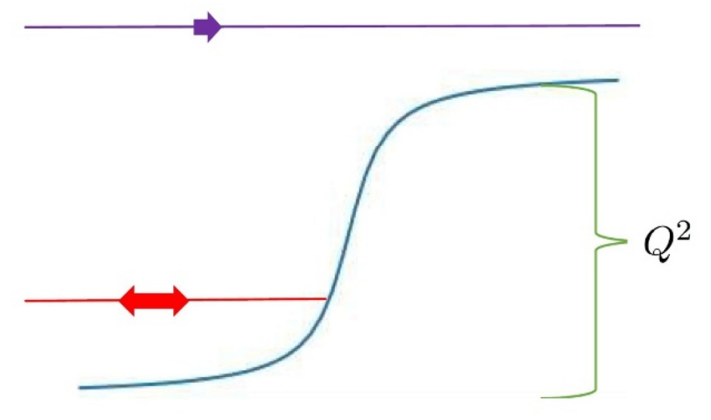

Figure 2. The potential experienced by GR-like modes in tortoise coordinates: the potential is monotonic with a mass gap that scales like $1 / k$. There are modes, marked in purple, that can escape to infinity, and modes, marked in red, that cannot. From the underlying $\operatorname{SL}(2, \mathbb{R})$ point of view they are different: the red modes arise from the principal discrete representations and are accompanied by a non-perturbative partner in the BH interior. The purple modes arise from the principal continuous representations and are not accompanied by a non-perturbative partner.

the contribution of the internal space, we shall thus obtain a physical dual, $W_{V}$, to each on-shell GR bound state $V$ in (5.4),

$$
W_{V}=e^{-\varphi-\bar{\varphi}} V_{\tilde{j} E}^{\omega=1} V_{\mathcal{N}} .
$$

More details of this construction are presented in appendix D.

Even without the detailed construction of $W_{V}$, it is clear that, much like $W$, (4.10), far from the $\mathrm{BH}$, the vertex operators $W_{V},(5.10)$, include an $\exp \left(\sqrt{\frac{k}{2}}\left(t_{L}-t_{R}\right)\right)$ factor. Hence, they are not mutually local with standard vertex operators, e.g. $V$ in (5.4), that include an $\exp \left(-i E\left(t_{L}+t_{R}\right)\right)$ factor, and which are clearly in the theory. To overcome this issue, we construct $F_{V}$, that is the Lorentzian analog of the fusion $F_{\ell} \sim W * W_{\ell}^{*}$ in the Euclidean case, (3.24); explicitly,

$$
F_{V}(w) \sim \int d^{2} z W^{+}(z) W_{V}^{*}(w)
$$

where the operator $W^{+}$is the one in eq. (4.10), and the operators $W_{V}$ are the GFZZ duals of $V$ discussed above, (5.10).

The operators $F_{V}$ are the non-perturbative completion of $V$. Each $V$ and its corresponding $F_{V}$ are two components of the same state in the theory. $V$ has a clear GR-like interpretation and $F_{V}$ does not; it is a stringy mode - an excitation of the folded string - that lives mainly behind the $\mathrm{BH}$ horizon. Just like $F$, the operators $F_{V}$ have a tail that falls rapidly outside the $\mathrm{BH}$.

There is something intriguing about this tail that reveals much of the physics associated with the folded string. Suppose that $V$ is an excitation that appears on the right wedge of the $\mathrm{BH}$ and not on the left wedge. Semi-classically, in GR, we can think about $V$ as a particle that starts its trajectory at the past singularity, crosses the past right horizon and falls to the future singularity after passing the future right horizon. 


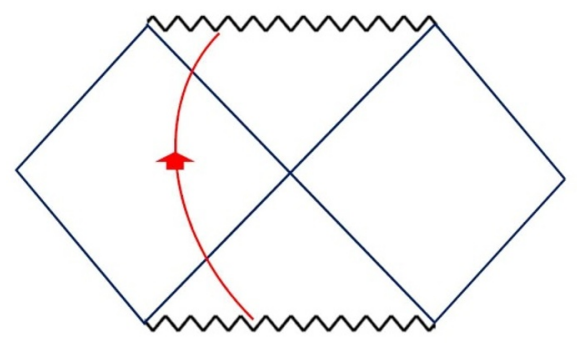

(a)

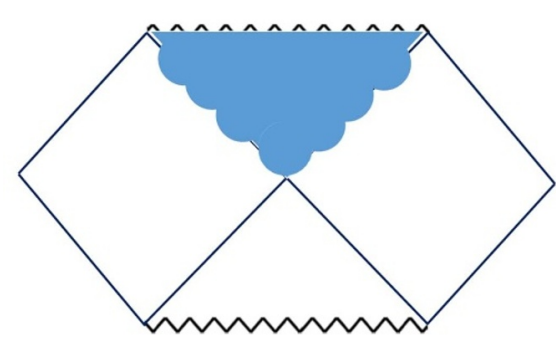

(b)

Figure 3. Folded strings and EPR: a particle that lives in the $\mathrm{BH}$ atmosphere on the left (a) is accompanied with a non-perturbative mode that lives on the BH-filling folded string (b). The folded string entangles the left and right sides by having tails on both.

Its companion, $F_{V}$, however, has an exponentially suppressed tail outside the horizon both in the right and left wedges (see figure 3). This follows from the symmetry of the interior filling folded string associated with the condensate $F$, and hence its bounded excitations. This is the sense in which the folded string entangles the left and right wedges of the $\mathrm{BH}$ and why it should be identified with EPR: the non-perturbative companion of a particle that propagates in the atmosphere of the right wedge has a tail also on the left wedge. ${ }^{20}$

While this follows from the $\mathrm{SL}(2, \mathbb{R})$ structure the question remains: how is this possible? What causes the information that semi-classically appears only on the left wedge to have (tiny) imprints on the left wedge? The answer to this was given in [33]. There, the semi-classical energy-momentum tensor associated with the folded string was calculated and it was claimed that if the number of folded strings scales like $1 / g_{0}^{2}$ then their backreaction is such that information that falls to the eternal $\mathrm{BH}$, say, from the right side, will not be able to cross the horizon, but rather will get smeared on the future right and left horizons. In [24], it was argued that the number of folded strings indeed scales like $1 / g_{0}^{2}$, since $F$ condenses on the sphere. The fact that $F_{V}$ has a tail also on the left wedge should be viewed as further evidence for these claims.

For this not to imply a violation of causality it is crucial that states in the continum representation are not accompanied with a non-perturbative partner. The reason is the following. The background before the condensation of $I$ is that of a linear dilaton with a strong coupling region. This background has a Little String Theory (LST) dual [67] that includes strings, but no gravity (as the string coupling constant vanishes at infinity). Much like in [68] we can think about the $\mathrm{SL}(2, \mathbb{R}) / \mathrm{U}(1)$ eternal black hole background as a thermofield double state that entangles between two LST's. Since the two LST's are decoupled an operator in one LST has to commute with an operator in the other LST.

At first it seems that figure 3 suggests otherwise as the GFZZ dual of the red line in that figure has a tail on both wedges. However, the red line in figure 3 (a) cannot be

\footnotetext{
${ }^{20} \mathrm{It}$ is important to recall the following. As discussed earlier, our finding gives rise to a realization of $\mathrm{ER}=\mathrm{EPR}$, but with a twist. This is one aspect of the twist, which is closely related to the other aspect that the BH interior does not appear to be empty, as it is filled with folded strings.
} 
described using only the left LST. It simply does not exsists in such a theory. It makes it appearence only after cutting off the strong coupling region via the entengelment to the other LST. States that do exsist in a single LST are the delta normalizable states. Such states do not have a GFZZ dual and so do not have a tail on both sides.

\section{Discussion}

The main point of the paper is to illustrate that in certain cases information in string theory can be quite different than in quantum field theories. In particular, each of the GR-like excitations that are bounded to the black fivebrane, in the sense that they live in the black-fivebrane atmosphere, is accompanied with a non-perturbative partner that lives on a folded string, which fills the black-fivebrane interior and has a tiny tail outside the horizon. The two combined form the exact state in the theory. It is the exactness of the underlying $\operatorname{SL}(2, \mathbb{R})$ CFT that allows us to pinpoint the non-perturbative partner in the case of black fivebranes. We believe, however, that the existence of a non-perturbative partner, which lives on an extended object in the $\mathrm{BH}$ interior, is likely to be generic in string theory.

We also think that this observation should play a key role in understanding the way information is extracted from black holes. However, we are still very much confused about the nature of this extraction; we are not even certain if it is gentle or brutal, in the sense discussed in the introduction. On the one hand, the identification of modes inside the $\mathrm{BH}$ with modes outside the $\mathrm{BH}$ is in the spirit of the $A=R_{B}$ and $\mathrm{ER}=\mathrm{EPR}$ proposals, which seems to suggest a gentle scenario. On the other hand, the non-perturbative partners do not propagate in an empty $\mathrm{BH}$ interior. Rather, they excite the folded strings that fill the $\mathrm{BH}$ interior. The existence of the folded strings seems to support a brutal scenario. In fact, their backreaction prevents information from falling into the $\mathrm{BH}$ in a rather non-trivial fashion [33]. It is as if they were designed for the brutal scenario.

It does feel a bit of a shame to have the key ingredient for the elusive gentle scenario and to end up with a brutal one. However, right now it is not clear to us how to evade this conclusion.

Perhaps the classical analysis of the folded strings [32, 33] is misleading, and it is the appropriate description only at low energies, of the order of the curvature $1 / \sqrt{k}$ (that are relevant for the discussion in the previous section)? Maybe above this energy, we should think about the folded string as a bound state of $k$ gravitons, in the spirit of $F=I^{k}$ discussed in subsection 3.5 (continued to the Lorentzian case)? At large $k$, such a bound state is likely to be close to threshold, from the point of view of an observer at infinity. In that case, a more energetic mode, with $E \gg 1 / \sqrt{k}$, should be sensitive to the fact that the folded string is made out of a $k$ replica of the ER bridge $I$.

\section{Acknowledgments}

We thank D. Kutasov for collaboration on some parts of this paper. This work is supported in part by a center of excellence supported by the Israel Science Foundation (grant number 2289/18) and BSF (grant number 2018068). 


\section{A $\quad \boldsymbol{I}_{\ell, \bar{\ell}}, \boldsymbol{W}_{\ell, \bar{\ell}}$ and $\boldsymbol{F}_{\ell, \bar{\ell}}$}

In this appendix, for completeness, we present the explicit form of generic $(\ell, \bar{\ell})$ excitations, discussed in section 3 , of the graviton condensate, $I$, the winding string condensate, $W$, and its fused condensate, $F$, in the worldsheet cigar CFT. Next, they are listed in turn.

First, from eq. (7.3) in [15], we have

$$
I_{\ell, \bar{\ell}}=P_{\ell}(\partial w, \ldots) P_{\bar{\ell}}(\bar{\partial} w, \ldots) e^{i \frac{p}{\sqrt{2 k_{b}}} x} e^{-\frac{1}{2} Q(\ell+\bar{\ell}) \phi},
$$

where $p$ is the angular momentum on the cigar (see appendix E),

$$
p=\bar{\ell}-\ell,
$$

and from eqs. (7.8) and (7.9) in $[15],{ }^{21}$ we have

$$
W_{\ell, \bar{\ell}}=e^{i \sqrt{\frac{2}{k_{b}}}\left(m x_{L}-\bar{m} x_{R}\right)-Q(\tilde{j}+1) \phi},
$$

where

$$
\tilde{j}+1=\frac{k_{b}-\ell-\bar{\ell}}{2}, \quad(m, \bar{m})=\frac{1}{2}\left(k_{b}+p, k_{b}-p\right) ;
$$

these are winding one operators with momentum $p$ around the cigar (see appendix E). Finally, the fusion of $W^{+} \equiv W_{1,1}$ with $W_{\ell, \bar{\ell}}^{*}$ is

$$
F_{\ell, \bar{\ell}}(w) \sim \int d^{2} z W^{+}(z) W_{\ell, \bar{\ell}}^{*}(w)
$$

normalized such that, for integer $k$,

$$
F_{\ell, \bar{\ell}}=P_{k+1-\ell}(\partial w, \ldots) P_{k+1-\bar{\ell}}(\bar{\partial} w, \ldots) e^{i \frac{p}{\sqrt{2 k_{b}}} x} e^{-\frac{1}{2} Q(2 k+2-\ell-\bar{\ell}) \phi} .
$$

В $\quad \beta^{\ell}=-P_{\ell}(\partial w, \ldots)$

In this appendix, we prove eq. (3.5) with (3.3). One way to do it is the following. The OPE of the left-moving piece of the winding condensate $W^{+}(z)$ with its conjugate $W^{-}(0)$, defined in eq. (3.9) with (3.3), is

$$
e^{-w(z)} e^{-\bar{w}(0)} \sim \frac{1}{z^{k+1}} e^{-[w(z)+\bar{w}(0)]}=\left.\sum_{n} \frac{z^{-1-k+n}}{n !}\left(\partial_{z}^{n} e^{-[w(z)+\bar{w}(0)]}\right)\right|_{z=0},
$$

hence, for integer $k$, the residue of the pole on the r.h.s. is

$$
-\frac{1}{k !} P_{k}(\partial w, \cdots) e^{-\frac{2}{Q} \phi},
$$

where

$$
P_{k}(\partial w, \cdots)=-\left(\partial^{k} e^{-w}\right) e^{w} .
$$

\footnotetext{
${ }^{21}$ Some sign conventions are different here.
} 
Now, since the operators $W^{ \pm}$arise from the left-moving piece of screening operators in the underlying $\mathrm{SL}(2, \mathbb{R})$ theory, a.k.a. truly marginal operators which are singlets of the $\mathrm{SL}(2, \mathbb{R})_{L} \times \mathrm{SL}(2, \mathbb{R})_{R}$ current algebra, the operator (B.2) must arise from a screening operator as well and, since we know the list of screening operators in the $\operatorname{SL}(2, \mathbb{R})$ theory, the only candidate is $\beta^{k} e^{-\frac{2}{Q} \phi}$, reduced to the Euclidean $\operatorname{SL}(2, \mathbb{R}) / \mathrm{U}(1)$ coset CFT and rewritten in the variables of the cigar, instead of the Wakimoto $\beta-\gamma$ variables in the underlying description (see e.g. [15], for a review). This is true for any integer $k$, and the normalization is set e.g. knowing that $\beta=\partial w$. All in all, this leads to (3.5) with (3.3).

\section{Folded string from $A d S_{3}$}

The main goal of this appendix is to describe the classical folded string in the $\mathrm{BH}$, from the point of view of $A d S_{3}$. The solutions are found via analytic continuation to the winding strings in the cigar. This gives further support to the stringy HH picture in figure 1 . We also give a geometrical interpretation to (4.8) and (4.9).

Lorentzian $A d S_{3}$ in global coordinates takes the form

$$
\frac{1}{k} d s^{2}=-\cosh ^{2}(\rho) d \tau^{2}+d \rho^{2}+\sinh ^{2}(\rho) d \theta^{2},
$$

where $0 \leq \rho<\infty,-\infty<\tau<\infty$ and $0<\theta \leq 2 \pi$. This coordinate system is useful when gauging $A d S_{3}$ to obtain the $\mathrm{SL}(2, \mathbb{R}) / \mathrm{U}(1)$ Euclidean cigar, since in that case we merely gauge the $\tau$ direction.

Another coordinate system, which is useful for us, covers the so-called Rindler-AdS space (see e.g. [69], for its definition and geometry). Lorentzian $A d S_{3}$ in Rindler coordinates takes the form

$$
\frac{1}{k} d s^{2}=-\sinh ^{2}(\rho) d t^{2}+d \rho^{2}+\cosh ^{2}(\rho) d x^{2},
$$

where $0 \leq \rho<\infty$, and $-\infty<t, x<\infty$. To obtain the $\mathrm{SL}(2, \mathbb{R}) / \mathrm{U}(1) \mathrm{BH}$, the $x$ direction has to be gauged. Equation (C.2) describes the region outside the BH. The horizon is at $\rho=0$. To cross the horizon, we take $\rho \rightarrow i \rho$, which gives

$$
\frac{1}{k} d s^{2}=\sin ^{2}(\rho) d t^{2}-d \rho^{2}+\cos ^{2}(\rho) d x^{2} .
$$

To see how (4.8) and (4.9) come about, we note that energy eigenstates behave like $V_{E} \sim \exp (-i E t)$, and $[66]$

$$
W^{ \pm} \sim \exp \left( \pm\left(\theta_{L}-\theta_{R}\right)\right)
$$

where $\theta$ is the angular direction in (C.1) and $t$ is the Rindler-AdS time direction. When $V_{E}$ goes around $W^{ \pm}$in the worldsheet then, because of (C.4), in target space it means that the wave function associated with $V_{E}$ makes a full circle, $\theta \rightarrow \theta+2 \pi$. Along this circle, it crosses the horizon 4 times. Each time the horizon is crossed, $t$ is shifted by $i \beta / 4$, and so by the time the circle is completed, $V_{E}$ acquires the Boltzmann factor, $\exp ( \pm \beta E)$, discussed in section 4.

Next, we turn to the classical folded string solution. This name is a bit misleading, since as discussed below, in $A d S_{3}$ the solution is not of a folded string. Only from the 


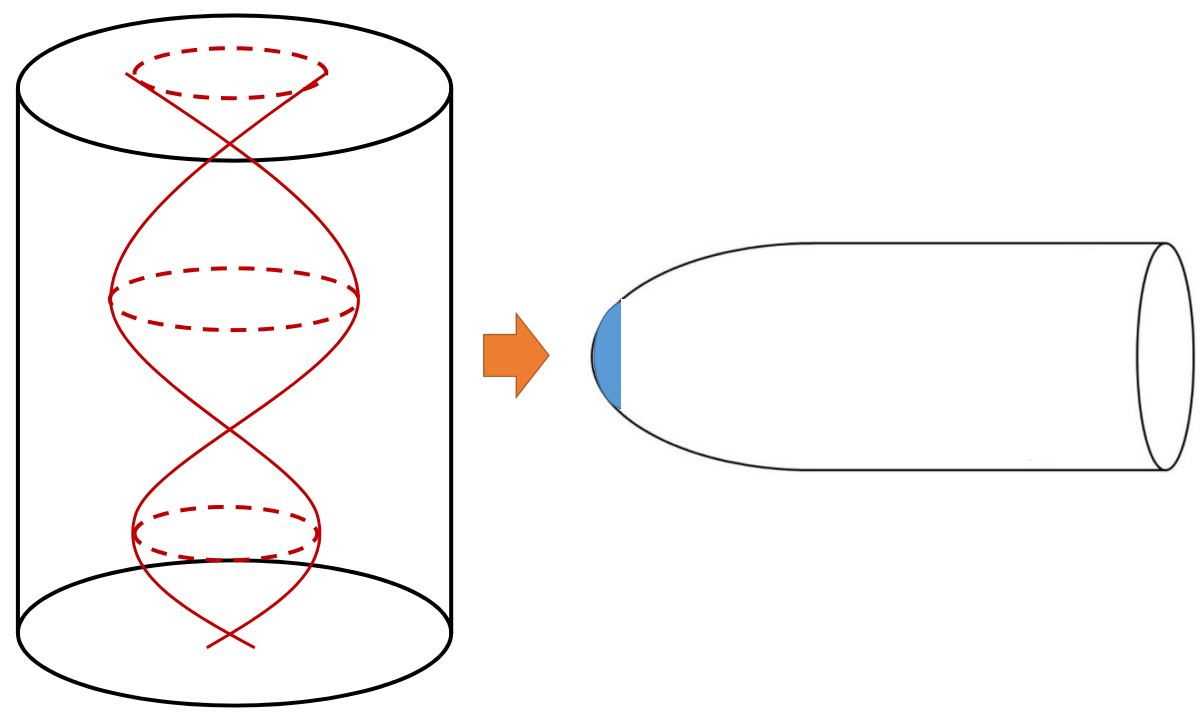

Figure 4. The pancake solution around the tip of the cigar (on the right) obtained, schematically, by squashing the short string solution in $A d S_{3}$ (on the left, [66]).

two-dimensional BH perspective, the solution is of a folded string. To find the solution, we first recall the classical short strings solutions, found in [66], that are relevant for the discrete states in the standard $J_{3}$ basis. The solutions take the form

$$
e^{i \theta} \sinh \rho=i e^{i \omega \tau_{1}} \sinh \rho_{0} \sin \left(\alpha \tau_{0}\right),
$$

and

$$
\tan \tau=\frac{\tan \left(\omega \tau_{0}\right)+\tan \left(\alpha \tau_{0}\right) / \cosh \left(\rho_{0}\right)}{1-\tan \left(\omega \tau_{0}\right) \tan \left(\alpha \tau_{0}\right) / \cosh \left(\rho_{0}\right)},
$$

where $\tau_{0}$ and $\tau_{1}$ are the timelike and spacelike worldsheet coordinates, respectively, $\omega$ is the winding number around $\theta$, and $\alpha$ and $\rho_{0}$ are parameters that describe the solution and are related to the quantum numbers associated with it, [66]. Since to obtain the $\operatorname{SL}(2, \mathbb{R}) / \mathrm{U}(1)$ Euclidean cigar, the $\tau$ direction is gauged away, the shape associated with this solution in the cigar is determined by (C.5) (and not by (C.6)). It takes the form of a pancake, with a center at the tip of the cigar. The size of the pancake is basically determined by $\rho_{0}$.

Note that, while the $A d S_{3}$ solution, (C.5) and (C.6), describes [66] a winding string that extends in time towards the $A d S_{3}$ boundary, till it reaches a maximal size, $\rho_{0}$, then contracts towards the center, and repeats to expand and contract periodically in time, the pancake solution, which is obtained, schematically, by squashing the one in $A d S_{3}$ in the time direction (see figure 4), is thus unoriented in the cigar background. This means, in particular, that at the classical level, one cannot distinguish between $W^{+}$and $W^{-}$. In fact, the pancake solution describes the Euclidean $F$, discussed in subsection 3.3.

We focus on the shortest short string, with $J_{L, R}^{a}=0$, which corresponds to $W$. It is obtained by taking $w= \pm 1, \alpha=\mp 1$, and $\rho_{0}=0 .{ }^{22}$ The size of the pancake in that case is zero: the pancake collapsed to a point.

\footnotetext{
${ }^{22}$ To derive this result, follow subsection 2.3 in [66].
} 


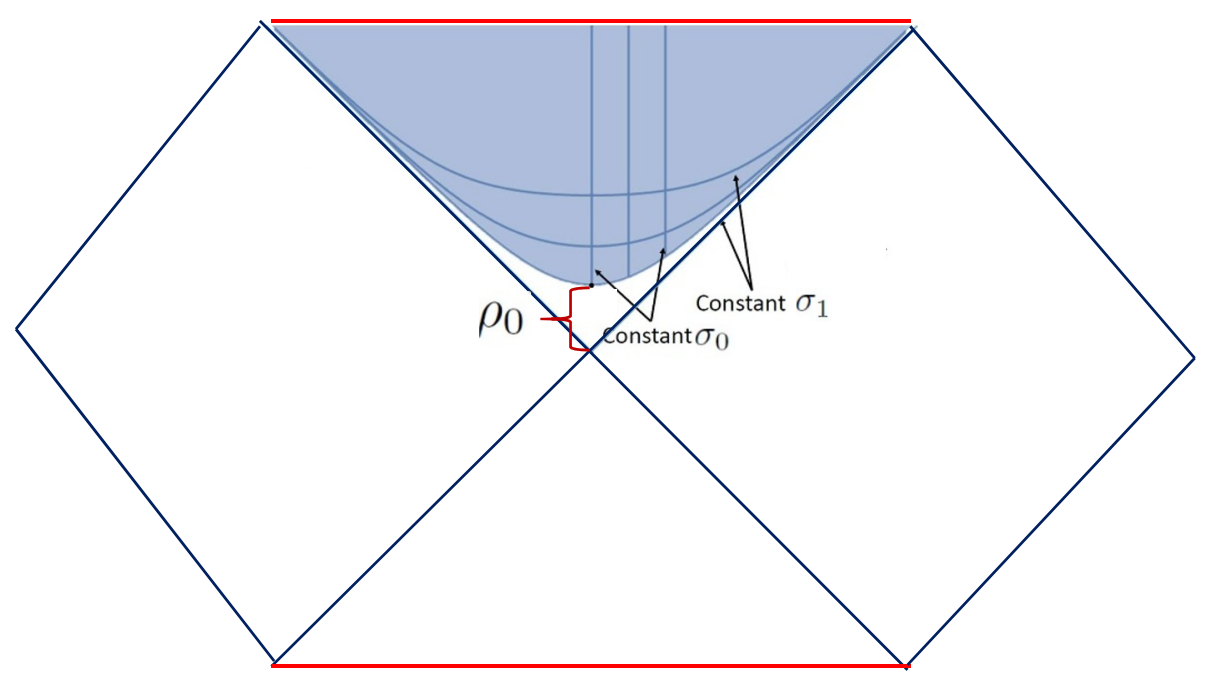

Figure 5. The solution (C.9), reduced on the BH geometry: both $\sigma_{0}$ and $\sigma_{1}$ run from $-\infty$ to $\infty$. They do so in a way that at $\rho=\rho_{0}$ (at the bottom of the shaded regime) and $t=i \beta / 4$ (namely, at $t=0$ in the $\mathrm{BH}$ interior), a closed folded string is created, propagates (in global time, namely, upwards) and ends at the singularity. The BH-filling string amounts to the $\rho_{0} \rightarrow 0$ limit.

To find the folded string solution, we note that (C.2) is obtained from (C.1) via double analytic continuation,

$$
\tau \rightarrow i x, \quad \theta \rightarrow i t
$$

Solutions in (C.2), or more precisely in (C.3), can thus be obtained from (C.5) via (C.7), combined with $\rho \rightarrow i \rho$.

Starting from (C.5) with $w=-\alpha=1$, we first shift $\tau_{0}$ by $\pi / 2$, then we double analytically continue on the worldsheet,

$$
\tau_{0} \rightarrow i \sigma_{1}, \quad \tau_{1} \rightarrow i \sigma_{0}
$$

and in the target space, (C.7), to get (after taking $\rho \rightarrow i \rho$ )

$$
t=\sigma_{0}, \quad \sin \rho=\sin \rho_{0} \cosh \sigma_{1},
$$

where $-\infty<\sigma_{0}, \sigma_{1}<\infty$.

Despite the fact that the range of the new space-like worldsheet coordinate is $-\infty<$ $\sigma_{1}<\infty$, (C.9) describes a closed string. From the $\mathrm{SL}(2, \mathbb{R}) / \mathrm{U}(1) \mathrm{BH}$ point of view, (C.9) is a closed folded string that is created behind the horizon, at $\rho=\rho_{0}$, and ends on the singularity; see figure 5. For $\rho_{0} \neq 0$, this is not a physical solution in the black fivebrane background, as it requires a contribution from internal space with a negative dimension. However, we care about the BH-filling solution with $\rho_{0}=0$, which is on-shell. In fact, it is invariant under the $\mathrm{SL}(2, \mathbb{R})_{L} \times \mathrm{SL}(2, \mathbb{R})_{R}$ affine symmetry, and so it is natural to identify it with $F$.

The reasoning of $[32,64]$ implies that there are many other on-shell solutions in the two-dimensional BH geometry. These, however, do not have specific quantum numbers under the $\mathrm{SL}(2, \mathbb{R})_{L} \times \mathrm{SL}(2, \mathbb{R})_{R}$, and so cannot be obtained from (C.5) and (C.6). 
From the $A d S_{3}$ point of view, the semiclassical configuration in eq. (C.9), unlike the one in eq. (C.5), ends on the boundary, and so in the dual space-time $C F T_{2}$ it does not correspond to a local operator. This is yet another aspect why $W$ is more fundamental than the $F$ condensate.

\section{The construction of $W_{V}$}

To obtain the GFZZ duals, $W_{V}$ in (5.10), of the GR bound states, $V$ in (5.4), it is convenient to write the non-compact, space-like $J_{2}$ eigenstates in the underlying $\operatorname{SL}(2, \mathbb{R})$ (the hyperpolic basis; see e.g. section 4 of [70]) in terms of the standard basis of the compact, time-like $J^{3}$ eigenstates,

$$
V_{j ; m_{2}}=\sum_{m_{3}} A_{m_{3}}\left(j, m_{2}\right) V_{j ; m_{3}},
$$

where here we restrict to the left-moving piece of $V_{j E}$, for simplicity, whose parent in $\mathrm{SL}(2, \mathbb{R})$ we denote by $V_{j ; m_{2}}$. For states in the $D_{j}^{+}$principal discrete representations (see e.g. [66] for definitions and details), (D.1) takes the explicit form

$$
V_{j ; m_{2}}=\sum_{m_{3}=j+1}^{\infty} A_{m_{3}}\left(j, m_{2}\right)\left(J_{0}^{+}\right)^{m_{3}-j-1} V_{j ; j+1} .
$$

Now, via the isomorphism between the principal discrete representation, $\hat{D}_{j}^{+, \omega=0}$, in affine $\mathrm{SL}(2, \mathbb{R})$, and its spectrally-flowed (a.k.a. twisted) $\hat{D}_{\tilde{j}=\frac{k}{2}-j-1}^{-, \omega=1}$ representation $[66],{ }^{23}$ we know that the operator

$$
\Phi_{\tilde{j} ; m_{2}}^{-, \omega=1}=\sum_{N=0}^{\infty} A_{j+1+N}\left(j, m_{2}\right)\left(J_{-1}^{+}\right)^{N} \Phi_{\tilde{j} ;-\tilde{j}-1}^{\omega=1}, \quad \tilde{j}=\frac{k}{2}-j-1,
$$

where $\Phi^{\omega}$ denotes a spectrally flowed, a.k.a. a twisted operator, in the $\omega$ sector (see [66] and [71] for details and notation), is related to (D.2) by GFZZ duality [15], and the two create the same state.

Finally, the reduction of these $J^{2}$ eigenstates to the Lorentzian $\operatorname{SL}(2, \mathbb{R}) / \mathrm{U}(1)$ coset, $V_{\tilde{j} E}^{\omega=1}$, is obtained by decomposing

$$
\Phi_{\tilde{j} ; m_{2}}^{-, \omega=1}=V_{\tilde{j} E}^{\omega=1} e^{i E x_{2}},
$$

where the energy $E$ is related to the $J^{2}$ eigenvalue $m_{2}$ by eq. (5.6), and $x_{2}$ is a canonically normalized scalar bosonizing the $J^{2}$ current,

$$
J^{2}=\frac{1}{Q} i \partial x_{2}
$$

Now, together with the ghosts and internal pieces, they give rise to the GFZZ duals, $W_{V}$, of the bound states $V$ in (5.4),

$$
W_{V}^{+}=e^{-\varphi-\bar{\varphi}} V_{\tilde{j} E}^{\omega=1} V_{\mathcal{N}}
$$

\footnotetext{
${ }^{23}$ We consider again the bosonic case here, for simplicity; the extension to the fermionic case is straightforward [15, 63].
} 
The conjugates, $W_{V}^{-}$, are obtained, similarly, starting with the $\hat{D}_{j}^{-}$principal discrete representations in (D.2).

A few comments on the properties of (D.3), (D.6) are in order:

- The energy eigenstates in the two-dimensional BH, which are reduced from their $\mathrm{SL}(2, \mathbb{R})$ parent, (D.3), consist of an infinite sum over string excitations, the $N^{\prime}$ th excitation contributing with its corresponding coefficient, $A_{j+1+N}\left(j, m_{2}\right)$.

- These $A_{m_{3}}\left(j, m_{2}\right) \equiv\left\langle j, m_{3} \mid j, m_{2}\right\rangle$, in eqs. (D.1)-(D.3), can be found in eq. (4.17) of [70], where the study of continuous bases for unitary irreducible representations of $\operatorname{SL}(2, \mathbb{R})$ is presented; note, in particular, that $\left\langle j, m_{2} \mid j, m_{2}^{\prime}\right\rangle=\delta\left(m_{2}-m_{2}^{\prime}\right)$, as expected for bound states in the $\mathrm{BH}$ zone.

- The wave functions of the stringy contribution to the bound states - the $W_{V}$ in eq. (D.6), behave like $e^{-\left(\frac{1}{Q}-Q j\right) \phi}$, at large radial $\phi$, as do the excitations of the winding string condensate in the Euclidean case - the $W_{\ell}$ in eq. (3.6), due to the $\Phi_{\tilde{j}=\frac{k}{2}-j-1}^{\omega=1}$ piece in eq. (D.3), and thus they are highly localized near the horizon, when the curvature and imaginary radial momentum are small, $Q, j / k \ll 1$, as opposed to their GFZZ duals, GR bound states - the $V$ in eq. (5.4), whose wave functions behave like $e^{-Q(j+1) \phi}$, instead, as do the excitations of the graviton condensate in the Euclidean case - the $I_{\ell}$ in eq. (3.4), and are thus spread over the BH atmosphere.

\section{E Some facts on $\operatorname{SL}(2, \mathbb{R}) / \mathrm{U}(1)$}

In this appendix, we collect some facts concerning properties of the $\operatorname{SL}(2, \mathbb{R}) / \mathrm{U}(1)$ quotient CFTs that are used in the text. The reader is referred to e.g. [15] for more facts and details.

Operators $V_{j}$ in $\operatorname{SL}(2, \mathbb{R}) / \mathrm{U}(1)$ quotient CFTs descend from operators $\Phi_{j}$ in the underlying $\operatorname{SL}(2, \mathbb{R})$ theory, and carry, in particular, the quantum number $j$ of the $\operatorname{SL}(2, \mathbb{R})$ representation, as well as other quantum numbers, depending on the particular choice of basis and subjected to the gauge condition imposed by the Abelian gauging done. The quantum number $j$ governs the radial dependence of the wave functions of states in the resulting sigma-model geometry. Concretely, the vertex operator $V_{j}$ decays at large $\phi$ as

$$
V_{j} \sim e^{-Q(j+1) \phi} .
$$

Stripping off the factor of the string coupling $e^{-Q \phi / 2},(2.10)$, that relates the vertex operator to the wave function, we find that the wave function of the state behaves at large $\phi$ as $e^{-Q(j+1 / 2) \phi}$, and hence it is normalizable if $j>-1 / 2$. However, unitarity of the CFT leads to a more restrictive bound on $j \in \mathbb{R}$ - the unitarity bound:

$$
-\frac{1}{2}<j<\frac{k-1}{2}
$$

where $k$ is the total level of the $\operatorname{SL}(2, \mathbb{R})$ theory,

$$
k=k_{b}-2=\sqrt{\frac{2}{Q}},
$$

and $k_{b}$ is the bosonic level. 
For instance, the cigar CFT is obtained by an axial gauging in the compact time-like direction of $\mathrm{SL}(2, \mathbb{R})$, and a convenient basis of states is thus eigenstates of the generators $\left(J^{3}, \bar{J}^{3}\right)$, whose eigenvalues are denoted by $(m, \bar{m})$. In the coset CFT, the latter are subject to the gauge condition

$$
\sqrt{\frac{2}{k_{b}}}(m,-\bar{m})=\left(p_{L}, p_{R}\right) \equiv\left(\frac{p}{R}+\frac{\omega R}{2}, \frac{p}{R}-\frac{\omega R}{2}\right), \quad R=\sqrt{2 k_{b}}, \quad p, \omega \in Z ;
$$

the integers $p$ and $w$ are thus the quantized momentum and winding, respectively, on the asymptotic cylinder of the cigar.

A large class of states in the cigar CFT is described by Virasoro primary vertex operators, $V_{j ; m, \bar{m}}$. Far from the tip of the cigar, they behave as

$$
V_{j ; m, \bar{m}} \simeq e^{i p_{L} x_{L}+i p_{R} x_{R}-Q(j+1) \phi},
$$

where $\left(p_{L}, p_{R}\right)$ is given in (E.4). The operators $W_{\ell, \bar{\ell}}$ in section 3 and appendix $\mathrm{A}$ are examples of such operators, with $\omega=1$ and $p=\bar{\ell}-\ell$. The operators $I_{\ell, \bar{\ell}}$ and $F_{\ell, \bar{\ell}}$ have non-zero oscillations modes on the cigar, the $P_{\ell}(\partial w, \ldots)$ and $P_{\bar{\ell}}(\bar{\partial} w, \ldots)$ in section 3 and appendices $\mathrm{A}$ and $\mathrm{B}$, on top of an (E.5) piece with $\omega=0$.

Open Access. This article is distributed under the terms of the Creative Commons Attribution License (CC-BY 4.0), which permits any use, distribution and reproduction in any medium, provided the original author(s) and source are credited.

\section{References}

[1] G. 't Hooft, On the quantum structure of a black hole, Nucl. Phys. B 256 (1985) 727 [INSPIRE].

[2] G. 't Hooft, The black hole interpretation of string theory, Nucl. Phys. B 335 (1990) 138 [INSPIRE].

[3] J.M. Maldacena, The Large $N$ limit of superconformal field theories and supergravity, Int. J. Theor. Phys. 38 (1999) 1113 [Adv. Theor. Math. Phys. 2 (1998) 231] [hep-th/9711200] [INSPIRE].

[4] L. Susskind, The transfer of entanglement: the case for firewalls, arXiv:1210.2098 [INSPIRE].

[5] K. Papadodimas and S. Raju, An infalling observer in AdS/CFT, JHEP 10 (2013) 212 [arXiv: 1211.6767] [INSPIRE].

[6] J. Maldacena and L. Susskind, Cool horizons for entangled black holes, Fortsch. Phys. 61 (2013) 781 [arXiv: 1306.0533] [INSPIRE].

[7] I. Bars and D. Nemeschansky, String propagation in backgrounds with curved space-time, Nucl. Phys. B 348 (1991) 89 [inSPIRE].

[8] S. Elitzur, A. Forge and E. Rabinovici, Some global aspects of string compactifications, Nucl. Phys. B 359 (1991) 581 [INSPIRE].

[9] G. Mandal, A.M. Sengupta and S.R. Wadia, Classical solutions of two-dimensional string theory, Mod. Phys. Lett. A 6 (1991) 1685 [inSPIRE]. 
[10] E. Witten, On string theory and black holes, Phys. Rev. D 44 (1991) 314 [INSPIRE].

[11] R. Dijkgraaf, H.L. Verlinde and E.P. Verlinde, String propagation in a black hole geometry, Nucl. Phys. B 371 (1992) 269 [inSPIRE].

[12] J. Teschner, Operator product expansion and factorization in the $H+(3)$ WZNW model, Nucl. Phys. B 571 (2000) 555 [hep-th/9906215] [InSPIRE].

[13] J. Troost, The non-compact elliptic genus: mock or modular, JHEP 06 (2010) 104 [arXiv: 1004.3649] [INSPIRE].

[14] A. Giveon, N. Itzhaki and D. Kutasov, Stringy horizons, JHEP 06 (2015) 064 [arXiv: 1502.03633] [INSPIRE].

[15] A. Giveon, N. Itzhaki and D. Kutasov, Stringy horizons II, JHEP 10 (2016) 157 [arXiv: 1603.05822] [INSPIRE].

[16] G. Giribet, Scattering of low lying states in the black hole atmosphere, Phys. Rev. D 94 (2016) 026008 [arXiv:1606.06919] [INSPIRE].

[17] G. Giribet, Stringy horizons and generalized FZZ duality in perturbation theory, JHEP 02 (2017) 069 [arXiv:1611.03945] [InSPIRE].

[18] N. Itzhaki and L. Liram, A stringy glimpse into the black hole horizon, JHEP 04 (2018) 018 [arXiv: 1801.04939] [INSPIRE].

[19] R. Ben-Israel, A. Giveon, N. Itzhaki and L. Liram, Stringy horizons and UV/IR mixing, JHEP 11 (2015) 164 [arXiv:1506.07323] [INSPIRE].

[20] R. Ben-Israel, A. Giveon, N. Itzhaki and L. Liram, On the black hole interior in string theory, JHEP 05 (2017) 094 [arXiv: 1702. 03583] [INSPIRE].

[21] V.A. Fateev, A.B. Zamolodchikov and Al.B. Zamolodchikov, unpublished.

[22] V. Kazakov, I.K. Kostov and D. Kutasov, A matrix model for the two-dimensional black hole, Nucl. Phys. B 622 (2002) 141 [hep-th/0101011] [InSPIRE].

[23] J.L. Karczmarek, J.M. Maldacena and A. Strominger, Black hole non-formation in the matrix model, JHEP 01 (2006) 039 [hep-th/0411174] [INSPIRE].

[24] A. Giveon and N. Itzhaki, Stringy black hole interiors, JHEP 11 (2019) 014 [arXiv: 1908.05000] [INSPIRE].

[25] M. Bershadsky and H. Ooguri, Hidden SL(n) symmetry in conformal field theories, Commun. Math. Phys. 126 (1989) 49 [INSPIRE].

[26] A. Gerasimov et al., Wess-Zumino-Witten model as a theory of free fields, Int. J. Mod. Phys. A 5 (1990) 2495 [INSPIRE].

[27] G. Giribet and C.A. Núñez, Aspects of the free field description of string theory on $A d S_{3}$, JHEP 06 (2000) 033 [hep-th/0006070] [INSPIRE].

[28] G. Giribet and C.A. Núnez, Correlators in AdS string theory, JHEP 06 (2001) 010 [hep-th/0105200] [INSPIRE].

[29] T. Fukuda and K. Hosomichi, Three point functions in sine-Liouville theory, JHEP 09 (2001) 003 [hep-th/0105217] [INSPIRE].

[30] A. Giveon and D. Kutasov, Notes on AdS $S_{3}$, Nucl. Phys. B 621 (2002) 303 [hep-th/0106004] [INSPIRE]. 
[31] J.B. Hartle and S.W. Hawking, Wave function of the Universe, Phys. Rev. D 28 (1983) 2960 [Adv. Ser. Astrophys. Cosmol. 3 (1987) 174] [InSPIRE].

[32] N. Itzhaki, Stringy instability inside the black hole, JHEP 10 (2018) 145 [arXiv: 1808.02259] [INSPIRE].

[33] K. Attali and N. Itzhaki, The averaged null energy condition and the black hole interior in string theory, Nucl. Phys. B 943 (2019) 114631 [arXiv:1811.12117] [INSPIRE].

[34] I. Bars and J. Schulze, Folded strings falling into a black hole, Phys. Rev. D 51 (1995) 1854 [hep-th/9405156] [INSPIRE].

[35] I. Bars, Folded strings in curved space-time, [hep-th/9411078].

[36] I. Bars, Folded strings, hep-th/9412044 [INSPIRE].

[37] A. Almheiri, D. Marolf, J. Polchinski and J. Sully, Black holes: complementarity or firewalls?, JHEP 02 (2013) 062 [arXiv:1207.3123] [INSPIRE].

[38] N. Itzhaki, Is the black hole complementarity principle really necessary?, [hep-th/9607028].

[39] S.L. Braunstein, S. Pirandola and K. Życzkowski, Better late than never: information retrieval from black holes, Phys. Rev. Lett. 110 (2013) 101301 [arXiv:0907.1190] [INSPIRE].

[40] S.D. Mathur, The information paradox: a pedagogical introduction, Class. Quant. Grav. 26 (2009) 224001 [arXiv:0909.1038] [INSPIRE].

[41] S.D. Mathur and D. Turton, Comments on black holes I: the possibility of complementarity, JHEP 01 (2014) 034 [arXiv: 1208. 2005] [INSPIRE].

[42] S.G. Avery, B.D. Chowdhury and A. Puhm, Unitarity and fuzzball complementarity: 'Alice fuzzes but may not even know it!', JHEP 09 (2013) 012 [arXiv:1210.6996] [INSPIRE].

[43] G. Giribet and A. Ranjbar, Screening stringy horizons, Eur. Phys. J. C 75 (2015) 490 [arXiv: 1504.05044] [INSPIRE].

[44] G. Dvali and C. Gomez, Black hole's quantum N-portrait, Fortsch. Phys. 61 (2013) 742 [arXiv:1112.3359] [INSPIRE].

[45] G. Dvali and C. Gomez, Black hole's 1/N hair, Phys. Lett. B 719 (2013) 419 [arXiv: 1203.6575] [INSPIRE].

[46] L. Susskind, Some speculations about black hole entropy in string theory, in The black hole, C. Teitelboim ed., World Scientific, Singapore (1998), hep-th/9309145.

[47] G.T. Horowitz and J. Polchinski, A correspondence principle for black holes and strings, Phys. Rev. D 55 (1997) 6189 [hep-th/9612146] [INSPIRE].

[48] A. Giveon, D. Kutasov, E. Rabinovici and A. Sever, Phases of quantum gravity in $A d S_{3}$ and linear dilaton backgrounds, Nucl. Phys. B 719 (2005) 3 [hep-th/0503121] [INSPIRE].

[49] D. Kutasov, Accelerating branes and the string/black hole transition, hep-th/0509170.

[50] A. Giveon and D. Kutasov, The charged black hole/string transition, JHEP 01 (2006) 120 [hep-th/0510211] [INSPIRE].

[51] G.T. Horowitz and A. Strominger, Black strings and P-branes, Nucl. Phys. B 360 (1991) 197 [INSPIRE].

[52] J.M. Maldacena and A. Strominger, Semiclassical decay of near extremal five-branes, JHEP 12 (1997) 008 [hep-th/9710014] [INSPIRE]. 
[53] A. Giveon, M. Porrati and E. Rabinovici, Target space duality in string theory, Phys. Rept. 244 (1994) 77 [hep-th/9401139] [INSPIRE].

[54] I. Bars and K. Sfetsos, Conformally exact metric and dilaton in string theory on curved space-time, Phys. Rev. D 46 (1992) 4510 [hep-th/9206006] [INSPIRE].

[55] A.A. Tseytlin, Conformal $\sigma$-models corresponding to gauged Wess-Zumino-Witten theories, Nucl. Phys. B 411 (1994) 509 [hep-th/9302083] [INSPIRE].

[56] S. Elitzur, O. Feinerman, A. Giveon and D. Tsabar, String theory on $A d S_{3} \times S^{3} \times S^{3} \times S^{1}$, Phys. Lett. B 449 (1999) 180 [hep-th/9811245] [INSPIRE].

[57] A. Giveon, D. Kutasov and O. Pelc, Holography for noncritical superstrings, JHEP 10 (1999) 035 [hep-th/9907178] [INSPIRE].

[58] O. Aharony, A. Giveon and D. Kutasov, LSZ in LST, Nucl. Phys. B 691 (2004) 3 [hep-th/0404016] [INSPIRE].

[59] N. Itzhaki, D. Kutasov and N. Seiberg, I-brane dynamics, JHEP 01 (2006) 119 [hep-th/0508025] [INSPIRE].

[60] A. Giveon, N. Itzhaki and J. Troost, Lessons on black holes from the elliptic genus, JHEP 04 (2014) 160 [arXiv:1401.3104] [INSPIRE].

[61] A. Giveon and N. Itzhaki, String theory at the tip of the cigar, JHEP 09 (2013) 079 [arXiv: 1305.4799] [INSPIRE].

[62] T.G. Mertens, H. Verschelde and V.I. Zakharov, Random walks in Rindler spacetime and string theory at the tip of the cigar, JHEP 03 (2014) 086 [arXiv:1307.3491] [INSPIRE].

[63] A. Giveon and N. Itzhaki, unpublished work.

[64] J.M. Maldacena, Long strings in two dimensional string theory and non-singlets in the matrix model, JHEP 09 (2005) 078 [hep-th/0503112] [INSPIRE].

[65] J. Polchinski, String theory. Volume 2: superstring theory and beyond, Cambridge University Press, Cambridge U.K. (1998).

[66] J.M. Maldacena and H. Ooguri, Strings in $A d S_{3}$ and $\mathrm{SL}(2, \mathbb{R})$ WZW model 1.: the spectrum, J. Math. Phys. 42 (2001) 2929 [hep-th/0001053] [INSPIRE].

[67] O. Aharony, M. Berkooz, D. Kutasov and N. Seiberg, Linear dilatons, NS five-branes and holography, JHEP 10 (1998) 004 [hep-th/9808149] [INSPIRE].

[68] J.M. Maldacena, Eternal black holes in Anti-de Sitter, JHEP 04 (2003) 021 [hep-th/0106112] [INSPIRE].

[69] M. Parikh and P. Samantray, Rindler-AdS/CFT, JHEP 10 (2018) 129 [arXiv:1211.7370] [INSPIRE].

[70] G. Lindblad and B. Nagel, Continuous bases for unitary irreducible representations of SU(1,1), Ann. I.H.P. Phys. Theor. 13 (1970) 27.

[71] R. Argurio, A. Giveon and A. Shomer, Superstrings on AdS $S_{3}$ and symmetric products, JHEP 12 (2000) 003 [hep-th/0009242] [INSPIRE]. 